\title{
Quantifying the impact of ecological memory on the dynamics of interacting communities
}

\author{
Moein Khalighi ${ }^{1 *}$, Didier Gonze ${ }^{2}$, Karoline Faust ${ }^{3}$, Guilhem Sommeria-Klein ${ }^{1}$, Leo Lahti ${ }^{1 *}$ \\ 1 Department of Computing, Faculty of Technology, University of Turku, Finland
}

2 Unité de Chronobiologie Théorique, Faculté des Sciences CP 231, Université Libre de Bruxelles,

Belgium

3 Laboratory of Molecular Bacteriology (Rega Institute), Department of Microbiology, Immunology and Transplantation, KU Leuven, Leuven, Belgium

\footnotetext{
* Corresponding authors

moein.khalighi@utu.fi (MKH)

leo.lahti@utu.fi (LL)
}

\section{Abstract}

Ecological memory refers to the influence of past events on the response of an ecosystem to exogenous or endogenous changes. Memory has been widely recognized as a key contributor to the dynamics of ecosystems and other complex systems, yet quantitative community models often ignore memory and its implications.

Recent studies have shown how interactions between community members can lead to the emergence of resilience and multistability under environmental perturbations. We demonstrate how memory can complement such models. We use the framework of fractional calculus to study how the outcomes of a well-characterized interaction model are affected by gradual increases in ecological memory under varying initial conditions, perturbations, and stochasticity.

Our results highlight the implications of memory on several key aspects of community dynamics. In general, memory slows down the overall dynamics and recovery times after perturbation, thus reducing the system's resilience. However, it simultaneously mitigates hysteresis and enhances the system's capacity to resist state shifts. Memory promotes long transient dynamics, such as long-standing oscillations and delayed regime shifts, and contributes to the emergence and persistence of alternative stable states. 
Collectively, these results highlight the fundamental role of memory on ecological communities and provide new quantitative tools to analyse its impact under varying conditions.

\section{Author summary}

An ecosystem is said to exhibit ecological memory when its future states do not only depend on its current state but also on its initial state and trajectory. Memory may arise through various mechanisms as organisms learn from experience, modify their living environment, collect resources, and develop innovative strategies for competition and cooperation. Despite its commonness in nature, ecological memory and its potential influence on ecosystem dynamics have been so far overlooked in many applied contexts. Here, we combine theory and simulations to investigate how memory can influence community dynamics, stability, and composition. We incorporate in particular memory effects in a multi-species model recently introduced to investigate alternative stable states in microbial communities, and assess the impact of memory on key aspects of model behavior. The approach we propose for modeling memory has the potential to be more broadly applied in microbiome research, thus improving our understanding of microbial community dynamics and ultimately our ability to predict, manipulate and experimentally design microbial ecosystems.

\section{Introduction}

The temporal variations observed in ecosystems arise from the interplay of complex deterministic and stochastic processes, the identification and characterization of which requires quantitative models. The empirical study of microbial communities provides an ideal source of data to inform the development of dynamical community models, since this active research area generates rich ecological time series under highly controlled experimental conditions and perturbations [1]. Nevertheless, despite the recent advances in metagenomic sequencing and other high-throughput profiling technologies that are now transforming the analysis of microbial communities [2], there has only been limited success in accurately modeling and predicting the complex dynamics in microbial communities. This highlights the need for re-evaluating and extending the available models to better account for the various mechanisms that underlie community dynamics $[1,3-7]$. One central shortcoming of the currently popular dynamical models is that they ignore the role of memory, that is, they are based on the assumption that the community's future behavior solely depends on its current state, perturbations, and stochasticity.

Ecological memory is present when the community's past states and trajectories influence its dynamics over extended periods. It is a fundamental aspect of natural communities, and its influence on community dynamics has been widely recognized across ecological systems [8-11]. Memory can emerge through a 
number of mechanisms, including the accumulation of abiotic and biotic material characterizing past legacies of the system $[12,13]$. Thus, developing and investigating new means to incorporate memory in dynamical models of ecological communities has the potential to yield more accurate mechanistic understanding and predictions.

Diverse approaches have been proposed to explore ecological memory, including time delays $[10,14,15]$, historical effects [16], exogenous memory [11], and buffering of disturbances [17]. A stochastic framework was used to evaluate the length, patterns, and strength of memory in ecological case studies [10]. However, the impact of memory has not been systematically addressed, and specific methods have been missing for incorporating memory into standard deterministic models of microbial community dynamics.

Potential community assembly mechanisms have been recently investigated based on extensions of the generalized Lotka-Volterra framework, which provides a standard model for species interactions [18-20]. The standard model has been extended by incorporating external perturbations [21], sequencing noise [22] and variance components [23], and to satisfy specific modeling constraints [24] such as compositionality [25] Generalized Lotka-Volterra models have also been combined with Bayesian Networks for improved longitudinal predictions [26]. One goal of these modeling efforts is to understand how the alternative community types reported in the human microbiome may arise, possibly in combination with external factors [27-31]. Despite the recent popularity of generalized Lotka-Volterra models in microbial ecology the impact of memory in these models has been largely ignored.

We address the above shortcomings by explicitly incorporating a class of memory effects into community interaction models using fractional calculus, which provides well-established tools for modeling memory $[32,33]$. We incorporate memory into a multi-species model that was recently used to illustrate the emergence of alternative states in microbial communities [18], and we then use this extended model to demonstrate how memory can influence critical aspects of community dynamics. This contributes to the growing body of quantitative techniques for studying community resistance, resilience, prolonged instability, transient dynamics, and abrupt regime shifts [34-38].

\section{2} 23

\section{Model}

The generalized Lotka-Volterra and its extensions are ordinary differential equation (ODE) systems. This class of models has been commonly used to model community dynamics, but their standard formulations ignore memory effects. Here, we show how ecological memory can be included in these models based on fractional calculus. This mathematical tool provides a principled framework for incorporating memory effects into ODE systems (see e.g. $[32,33,39,40]$ ), thus allowing a systematic analysis and quantification 47 of memory effects in commonly used dynamical models of ecological communities. 


\section{a Community model}
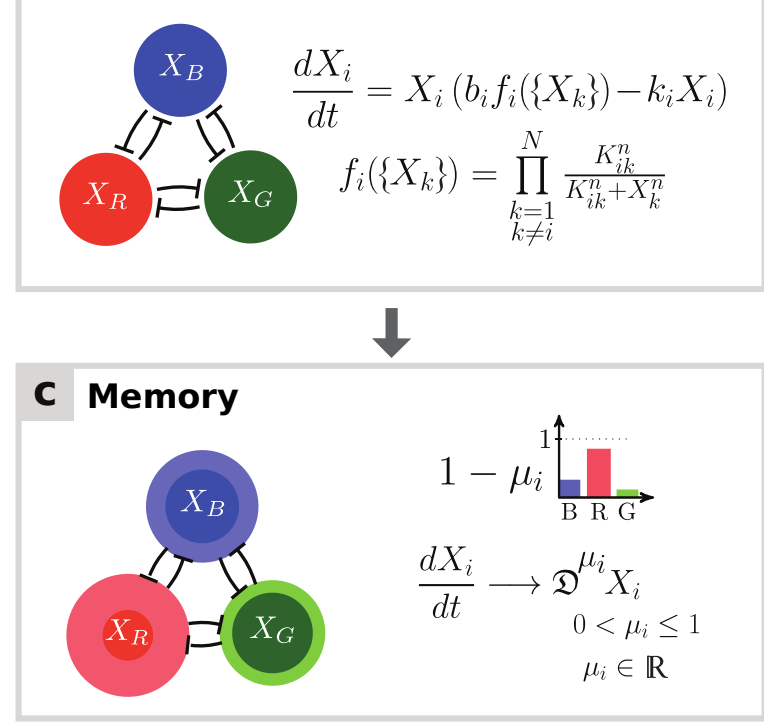

\section{b Perturbation}
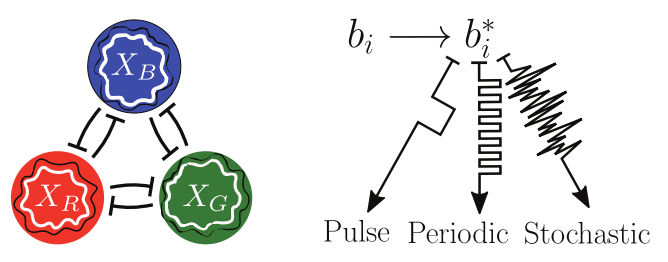

\section{d Perturbation + Memory}

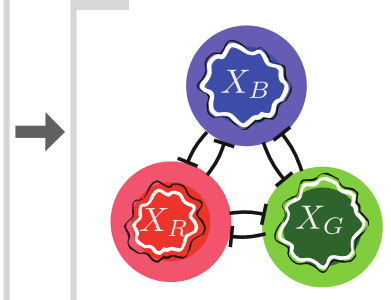

Modified

dynamical

properties

Fig 1. Schematic illustration of a three-species community in the presence of memory and perturbations. (a) The mutual interaction model describes the dynamics of species abundances $X_{i}$, which depends on the growth rates $b_{i}$, death rates $k_{i}$, and inhibition functions $f_{i}$, where $K_{i j}$ and $n$ denote interaction constants and Hill coefficients, respectively [18]. (b) Standard perturbations include pulse, periodic, and stochastic variation in species immigration, death, or growth rates. Such perturbations may trigger shifts between alternative states. (c) Memory (bolded circles) can be incorporated into dynamical models by substituting the integer-order derivatives with fractional derivatives $\mathfrak{D}^{\mu_{i}}$ of order $\mu_{i}$ (see [32] and Appendix S1). As decreasing $\mu_{i}$ values correspond to increasing memory, memory is measured as $1-\mu_{i}$. When all community members have the same memory $\left(\mu_{i}=\mu\right.$ for all $\left.i\right)$, the system is said to have commensurate memory, otherwise incommensurate. Increasing memory changes community dynamics, in particular by slowing it down and modifying the stability landscape around stable states. (d) Ecological memory can change the system's dynamics under perturbation.

Let us first consider a simple community with three species that tend to inhibit each other's growth

(Fig 1a). We will later extend this model community to a larger number of species. To model this system, we employ a non-linear extension of the generalized Lotka-Volterra model that was recently used to demonstrate possible mechanisms underlying the emergence of alternative states in a multi-species community [18]. This non-linear model describes the dynamics of a species $i$ as a function of its growth rate, death rate, and an interaction term determined by the interaction matrix between all species pairs, 54 as described in Fig 1a. Under certain conditions, this model gives rise to a tristable community, where each stable state corresponds to the dominance of a different species. The community can shift from one stable state to another following an external or internal perturbation (Fig 1b).

To introduce memory, we extend this model by incorporating fractional derivatives. In this extended formulation, the classical derivative operator $d / d t$ is replaced by the fractional derivative operator $\mathfrak{D}^{\mu_{i}}, \quad 59$ where $\left.\left.\mu_{i} \in\right] 0,1\right]$ is the non-integer derivative order for species $i$ (Fig 1c). The fractional derivative is 
bioRxiv preprint doi: https://doi.org/10.1101/2021.09.01.458486; this version posted September 2, 2021. The copyright holder for this preprint (which was not certified by peer review) is the author/funder, who has granted bioRxiv a license to display the preprint in perpetuity. It is made available under aCC-BY 4.0 International license.

species $i$ [32]. The strength of memory for species $i$ is measured as $1-\mu_{i}$. The three special cases of this model include (i) no memory $\left(\mu_{i}=\mu=1\right.$ for all species $i$ ), which corresponds to the original community model in [18]; (ii) commensurate memory, where all species have equal memory $\left(\mu_{i}=\mu \leq 1\right)$; and (iii) incommensurate memory, where $\mu_{i}$ may be unique for each $i$, and hence the degree of memory may differ between species. We numerically solve the fractional-order model with varying values of the parameter $\mu_{i}$, thus inducing varying levels of memory, and use it to analyse the effect of memory on various aspects of community dynamics, in particular its response to perturbations (Fig 1d).

Pulse perturbation

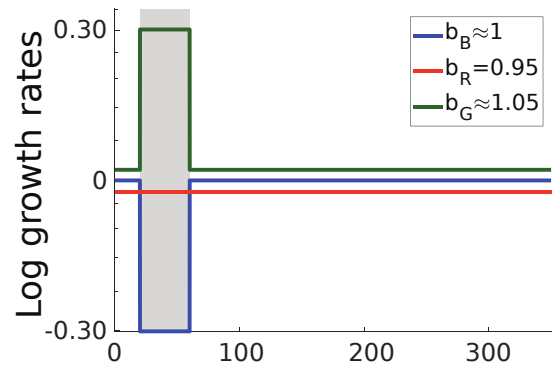

b

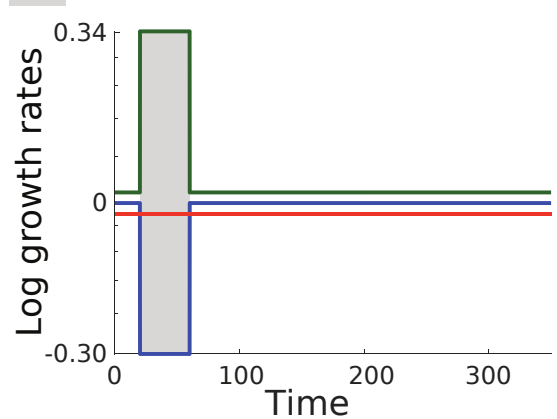

Community dynamic
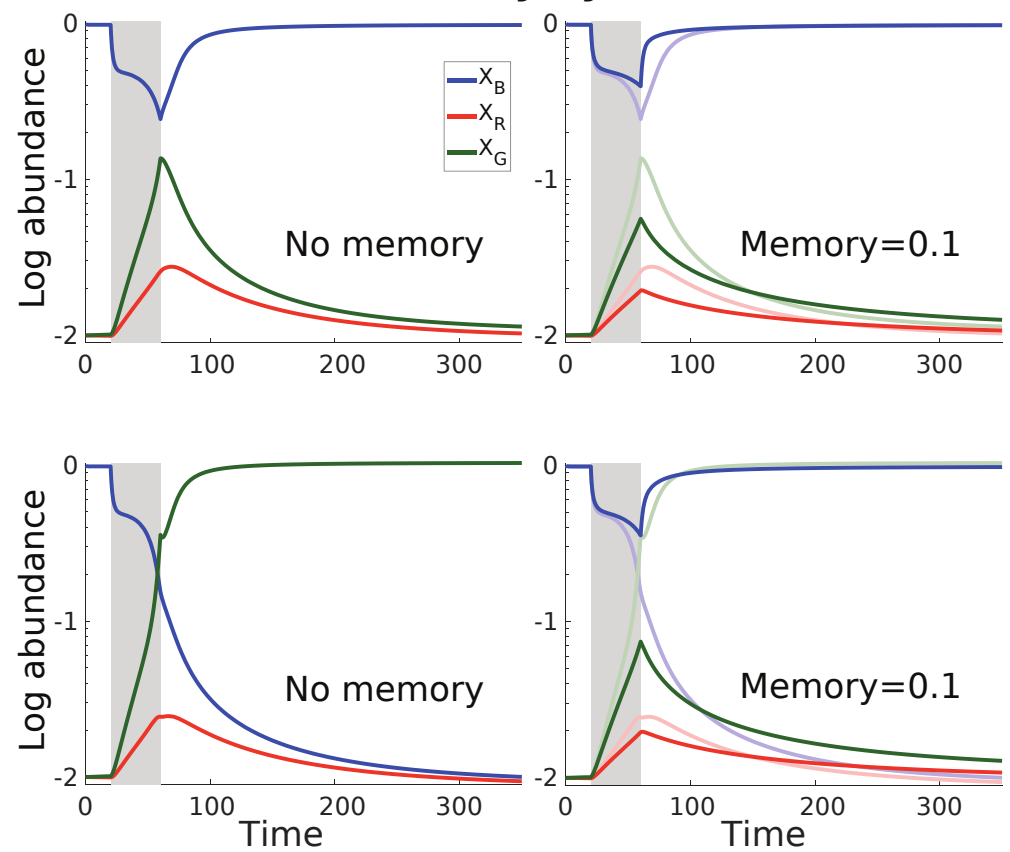

Fig 2. Impact of commensurate memory on community resistance and resilience. (a) A pulse perturbation is applied to the community (left panel): the growth rate of the blue species is lowered while that of the green species is simultaneously raised. The perturbation temporarily moves the community away from its initial stable state, characterized by blue species dominance (middle panel). Introducing commensurate memory (right panel) increases resistance to perturbation since the community is not displaced as far from its initial state compared to the memoryless case (shown in superimposition). The effect on resilience depends on the time scale considered: while memory initially hastens the recovery after the perturbation, it slows down the later stages of the recovery (starting around the time step 150). (b) A slightly stronger pulse perturbation is applied (left panel), triggering a shift toward an alternative stable state dominated by the green species (middle panel). Memory can prevent the state shift (right panel). Thus, here, not only does memory increase community resistance to perturbation, but also resilience as manifested by the prevented state shift.

\section{Results}

We have shown above how ecological memory can be incorporated in dynamical community models based on the framework of fractional calculus. Next, we use numerical simulations and analyses of this model to highlight the impact of memory on key dynamical properties of multi-species communities. 
In general, memory adds a certain inertia in community dynamics as the influence of past states gradually fades out. Commensurate memory thus slows down the overall dynamics, which may lead to qualitative changes in the dynamics as well as community composition under certain conditions. In initial conditions. In the first section below, we report the consequences of these changes on community resistance and resilience.

The emergence of alternative community states has been debated in the microbiome research literature. For instance, [18] demonstrated how pulse perturbations can bring the 3 -species system to a boundary of the tristability region, which then triggers a transition to an alternative stable state. In that model, such a transition can be for instance controlled by changes in the species' growth rates. In the second section below, we report how memory can exert additional influence on the resulting dynamics and alter the community's stability landscape.

\section{Resistance and resilience}

Resistance refers to a system's capacity to withstand a perturbation without changing its state, while resilience refers to its capacity to recover to its original state after a perturbation [41]. To examine the perturbed the system by changing the species growth rates over time. Specifically, we investigated the three-species community under pulse (Figs 2), periodic (Fig 3), and stochastic (Figs 4) perturbations, 94 and analysed the impact of these three types of perturbations on community dynamics in the presence of 95 memory, which is commensurate in this subsection.

Our results show that memory tends to increase resistance to perturbations by allowing the competing species' coexistence for a longer time. In the presence of memory, switches between alternative community states take place more slowly following a pulse perturbation (Fig 2a), or in some cases may be prevented entirely (Fig 2b). Fig S1 provides a further example of the increased resistance provided by memory in a larger, unstructured community, where memory helps preserve the stable state after a pulse perturbation compared to the corresponding memoryless system.

After the perturbation has ceased, memory initially hastens the return to the original state, but then slows it down in the later stages of the recovery (Fig 2a). Thus, the impact of memory on resilience 104 is multi-faceted: depending on the time scale considered, memory may either slow down or hasten the 105 recovery from perturbations, thus reducing or increasing resilience. Furthermore, in multistable systems, 106 
bioRxiv preprint doi: https://doi.org/10.1101/2021.09.01.458486; this version posted September 2, 2021. The copyright holder for this preprint (which was not certified by peer review) is the author/funder, who has granted bioRxiv a license to display the preprint in perpetuity. It is made available under aCC-BY 4.0 International license.

a Successive pulse perturbations

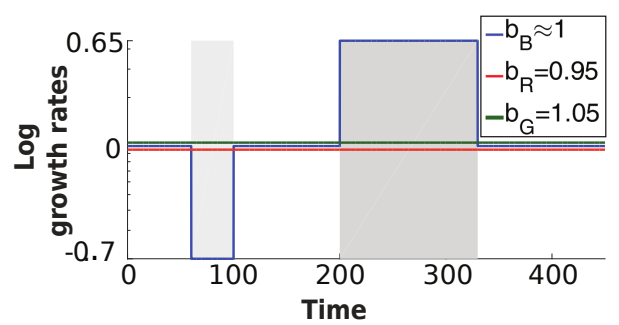

b Dynamical behavior

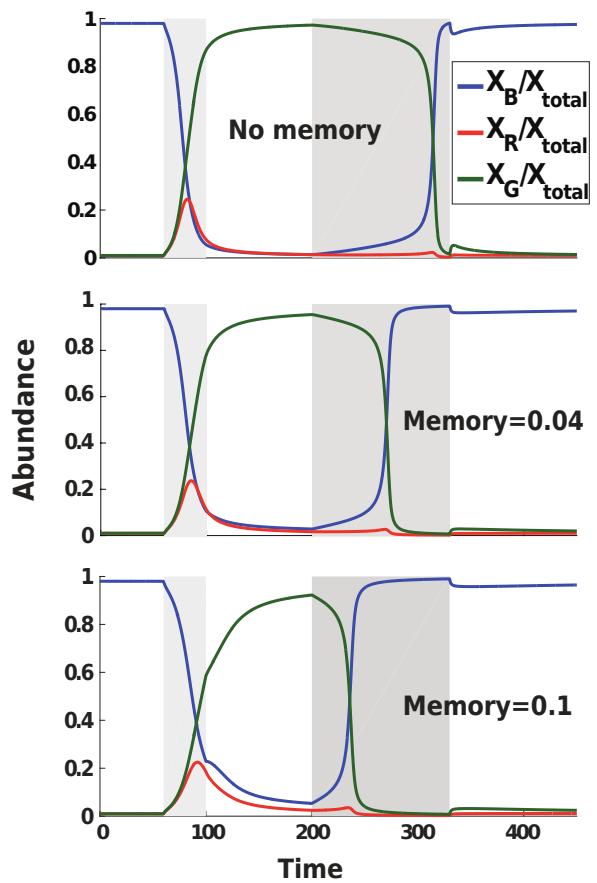

C Periodic perturbation

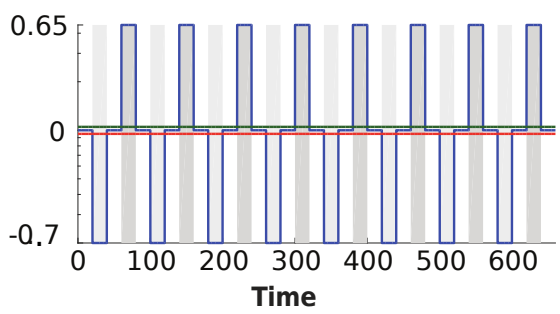

d Dynamical behavior
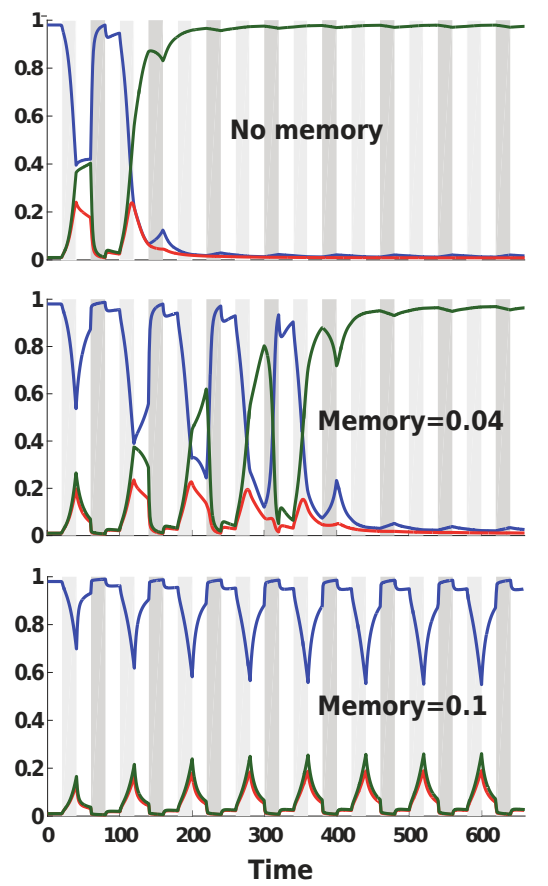

Fig 3. Multi-pulse and periodic perturbations: memory impact on hysteresis and transient oscillations. (a) Two opposite pulse perturbations are applied successively: the blue species growth rate is first briefly lowered, and then raised for a longer time. (b) The top panel shows the hysteresis in the system: the state shift towards the dominance of the green species occurs faster after the first perturbation than the shift back to the initial stable state after the second perturbation. Introducing commensurate memory (middle and bottom panels) delays the first state shift, thus increasing resistance, and hastens the second state shift, thus mitigating the hysteresis effect and increasing long-term resilience. (c) Rapidly alternating opposite perturbations are applied to the blue species growth rate with a regular frequency. (d) Without memory (top), the hysteresis effect leads to a permanent shift towards the green-dominated alternative stable state after a few oscillations. Adding commensurate memory mitigates the hysteresis, thus extending the transitory period (middle), which may generate longstanding oscillations in community composition before the community converges to a stable state (bottom). 
memory may enhance resilience by promoting the persistence of the original stable state (Fig $2 \mathrm{~b}$ ).

Considering two successive pulse perturbations in opposite directions highlights another way memory can affect resilience in multistable systems (Fig 3a). After a state shift triggered by a first perturbation, 109 memory may hasten recovery to the initial state following a second, opposite perturbation, hence increasing 110 long-term resilience. Memory can thus mitigate the hysteresis that is typical of many ecological systems. 111

In the presence of regularly alternating opposite pulse perturbations, akin to those experienced by 112 marine plankton or the gut microbiome, the community may not be able to recover its initial state if the 113 perturbations follow each other too rapidly. In such circumstances, memoryless communities reach a new 114 stable state faster than the communities with memory, as the latter resist the perturbations for a longer 115 time due to the reduced hysteresis (Fig 3b). This may lead to community dynamics being trapped in 116 long-lasting transient oscillations.

Finally, we analyse the role of stochastic perturbations, which are an essential component of variation 118 in real systems. Under stochastic perturbation (Fig 4a), ecological memory can dampen the fluctuations 119 and significantly delay the shift towards an alternative stable state (Fig 4b). This demonstrates in a more 120 realistic perturbation setting how memory can promote community resistance.

Memory can nevertheless have unexpected effects on community dynamics when its strength is tuned 122 to bring the system in the vicinity of the tristable region, where the outcome of the dynamics is highly 123 sensitive to initial conditions (Fig 4c). Under such conditions, minute changes in memory can push the 124 system over a tipping point towards another attractor, radically changing the outcome. This illustrates 125 that, beyond slowing down the dynamics and damping perturbations, memory can have non-trivial effects 126 on the system's stability landscape, which we investigate in the next section.

\section{Impact on stability landscape}

Let us now consider a more complex community of 15 species structured into three groups through their 129 interaction matrix. Each of these groups represents a set of weakly competing species-e.g., due to 130 cross-feeding interactions that mitigate competition, while species belonging to different groups compete 131 more strongly with each other (Fig 5a). We show that adding incommensurate memory in such a system 132 can change the final stable state of the community even in the absence of perturbation. In particular, 133 increasing the strength of memory in the group that is dominant in the stable state of the memoryless 134 system can lead to its exclusion from the new stable state (Fig 5b-c). Around the threshold value, long 135 transients can be observed (Fig 5d): even without changing any of the model parameters or imposing 136 noise, an abrupt regime shift is triggered by the accumulated effect of memory after a long period of 137 subtle, gradual changes.

Remarkably, adding memory in a given species may lead to either a reduction or an increase in its 139 
a Stochastic perturbation

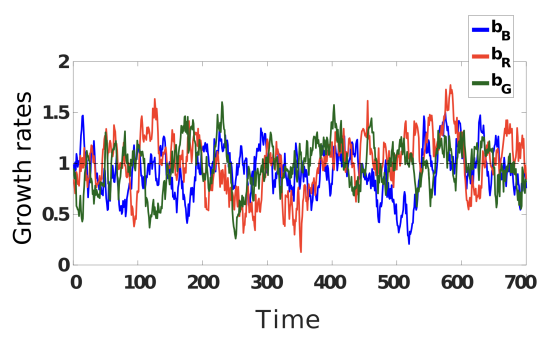

b Dynamical behavior

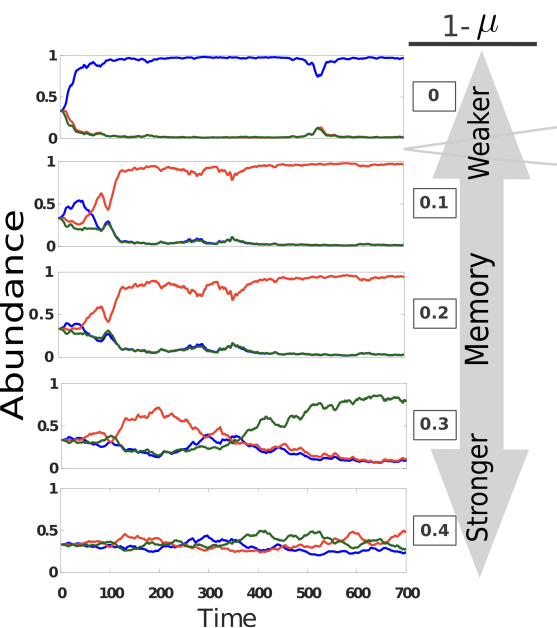

\section{Particular cases}

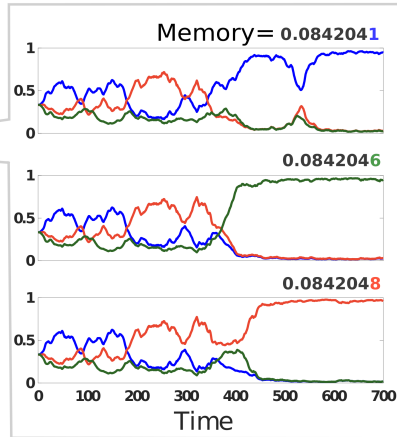

Fig 4. Stochastic perturbations with commensurate memory effects. (a) Stochastic perturbation in a three-species system: species growth rates $b_{i}$ vary stochastically through time according to an Ornstein-Uhlenbeck process (see Appendix S2). (b) Dynamical behavior of the system in response to the stochastic perturbation for equal initial species abundances and varying memory level: in addition to slowing down community dynamics, increasing memory limits the overall variation in species abundances, thus enhancing the overall resistance of the system. (c) For some memory strengths, the final state of the system can be sensitive to slight variations in memory, with drastic consequences on community composition.

abundance depending on the conditions. While Fig 5b-c illustrates the exclusion of a group of species 140 with higher memory from the stable state in the absence of perturbation, memory may conversely increase 141 the persistence or abundance of a species in the presence of stochastic perturbation (Fig S2b). In fact, in 142 the presence of perturbation, the dominance of any of the species may be achieved by tuning memory in a 143 single species. This result holds both in the case of pulse (Fig S2a) and stochastic (Fig S2b) perturbation. 144

Bifurcation diagrams further show that, in addition to modifying the boundary between stable states 145 in the space of initial conditions, memory can also broaden the region of the model's parameter space 146 that exhibits multistability (Fig S3). We illustrate for instance in Figure 6 that incommensurate memory 147 can induce multistability in a 3 -species community that would otherwise converge to a single stable 148 state in the absence of memory. Ecological memory thus provides an alternative and largely overlooked 149 mechanism for the emergence of multistability.

Finally, we show that simply setting similar levels of ecological memory within groups of species in an 151 otherwise unstructured community may lead to the formation of coherent species assemblages with shared 152 dynamics (Fig S4). This provides an additional mechanism for the emergence of distinct community 153 types, each associated with the dominance of one such assemblage. Hence, our results show that memory 154 can by itself lead to the emergence of alternative community types, between which the community may 155 switch following a change in either initial conditions (Fig 6) or memory strength (Fig S4). 
bioRxiv preprint doi: https://doi.org/10.1101/2021.09.01.458486; this version posted September 2, 2021. The copyright holder for this

a

15 Species in 3 groups

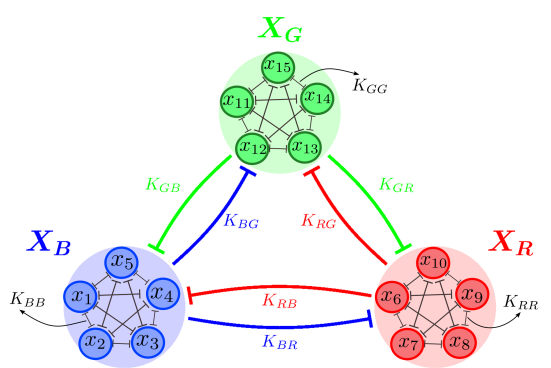

Interaction matrix $\mathbf{K}$

$K_{i j} X_{B} X_{R} X_{G}$

$X_{B} K_{B B}\left|K_{B R}\right| K_{B C}$

$X_{R} K_{R B} K_{R R} K_{R C}$

\begin{tabular}{l|l|l|l|l}
$X_{G}$ & $K_{G B}$ & $K_{G R}$ & $K_{G G}$ \\
\hline
\end{tabular}

d

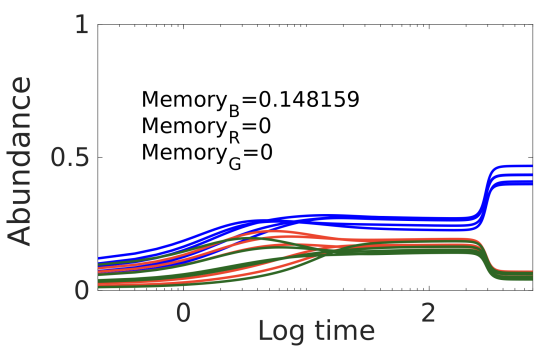

b
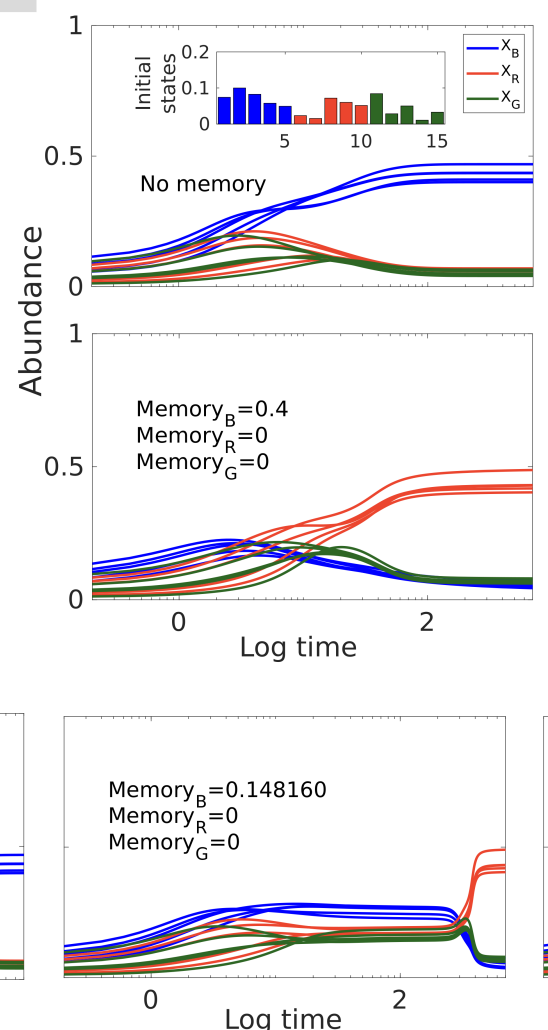

C
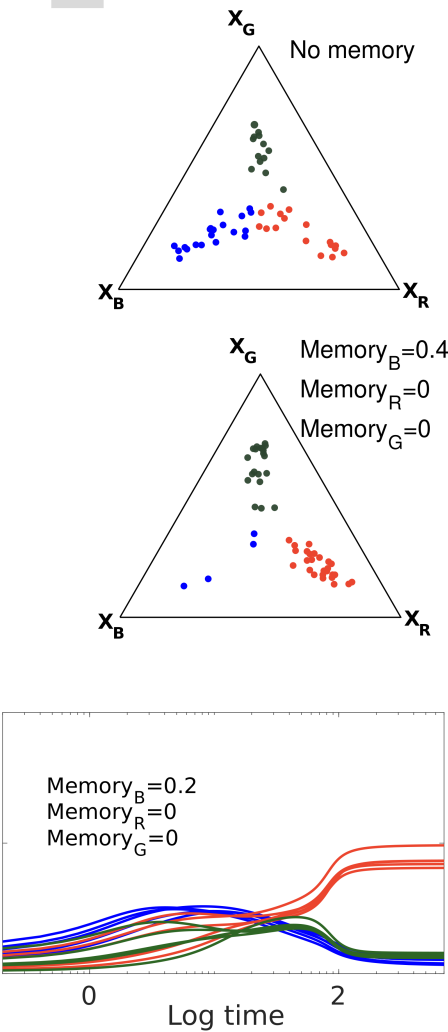

Fig 5. Impact of incommensurate memory on the community stability landscape: regime shifts without perturbation. (a) A simulated mutual interaction model with 15 species in three groups, blue, red, and green (see [18]). The interactions between species from different groups and within each group are illustrated. The within-group interactions are stronger than between-group interactions. (b) Starting from random initial conditions, the blue group of species dominates the community at the stable state when no memory is present (top). Imposing memory on the blue species leads to a temporary rise in abundance, but ultimately another (red) group of species dominates instead (bottom). (c) The stable state distributions of 50 simulations are represented by ternary plots. Each dot shows, for one simulation, the identity of the dominant group (color) and the average relative abundances of the three groups (position in the triangle) at convergence time (see Appendix S2 for details). In the memoryless system (top), the three groups roughly have the same chance of dominating the stable state, whereas imposing memory effects on the blue set of species (bottom) favors stable states where those species are not dominant. (d) Exceeding a particular threshold on incommensurate memory on the blue species (here, 0.14816) leads to an abrupt regime shift after a long period of subtle, gradual inclines, without changing any model parameters or adding noise.

\section{Discussion}

Our understanding of ecological community dynamics heavily relies on mathematical modeling. Dynamical 158 community modeling is a particularly active research area in microbial ecology, where recent studies have 159 proposed numerous mechanistic models of microbial community dynamics exploring the role of interactions, 160 stochasticity, and external factors $[1,18,42-44]$. These studies have, however, largely neglected the role of 161 ecological memory despite its potentially remarkable impact on community variation.

We have shown how ecological memory can be incorporated into models of microbial community 


\section{a No Memory}

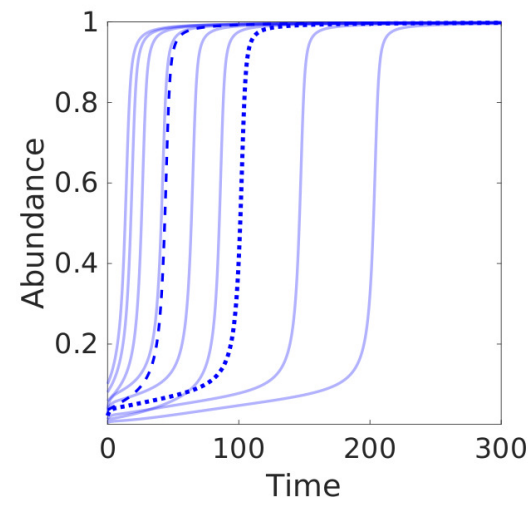

\section{b Memory}

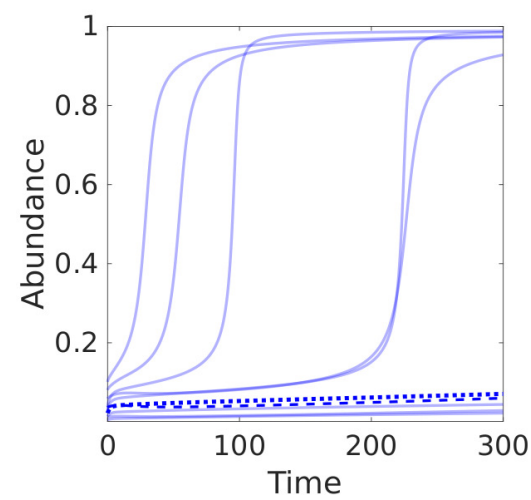

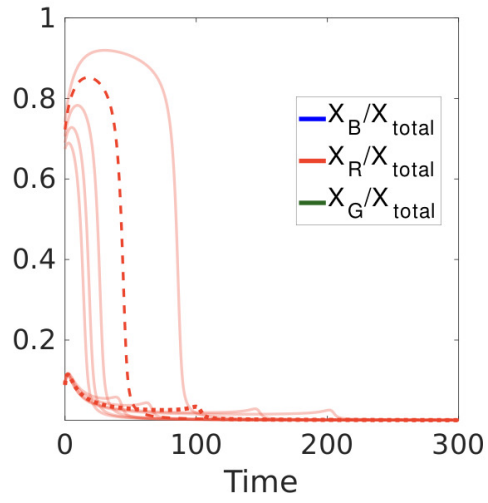
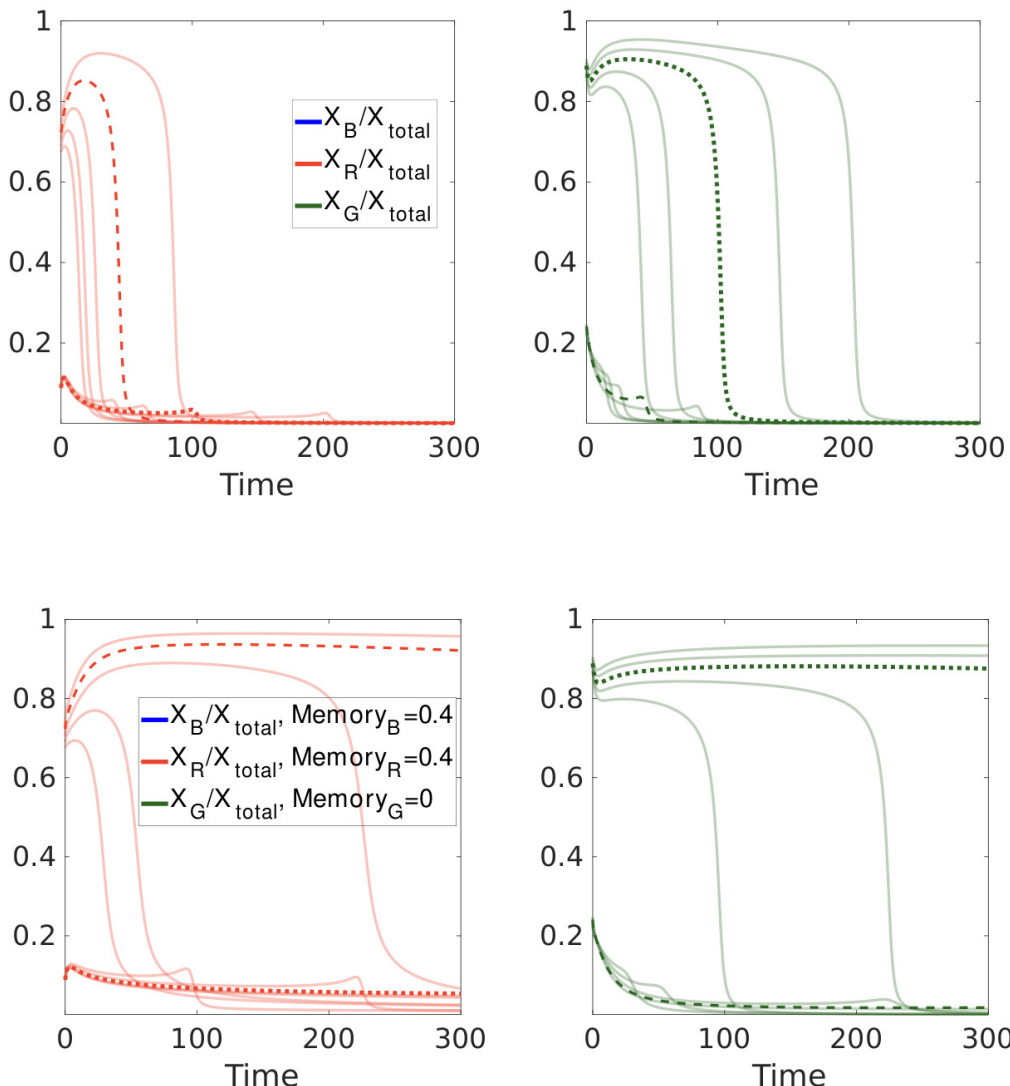

Fig 6. Incommensurate memory can induce multistability. Three-species community model that converges: (a) in the absence of memory, to a single stable state irrespective of initial abundances (see Fig S3), and (b) in the presence of incommensurate memory, to different stable states depending on species initial abundances. In each row, the three panels show the relative abundances of the blue, red and green species along time for the same set of initial conditions. In (b), the dashed and doted lines indicate the initial abundance thresholds that separate the three alternative attractor states, each corresponding to the dominance of a different species. No change in stable state is observed for the same values in the corresponding memoryless system (a).

dynamics, and used this modeling tool to demonstrate the role of memory as a potential key determinant 164 of community dynamics. This has allowed us to expand our understanding of the impact of memory on 165 community response to perturbation, long transient dynamics, delayed regime shifts, and the emergence 166 of alternative community states.

Ecological communities are constantly subject to perturbations arising from external factors, as well 168 as from internal processes and interactions between community members. Environmental fluctuations 169 through time have a fundamental influence on ecological communities: they may promote species 170 coexistence, increase community diversity $[45,46]$, contribute to the properties of stable states $[37,47]$, and 171 in some cases, facilitate abrupt regime shifts [47]. Our analysis of memory in perturbed communities is 172 closely linked to recent studies analysing the response of experimental microbial communities to antibiotic 173 pulse perturbation $[48,49]$, or the impact of periodic perturbations on the evolution of antimicrobial 174 
resistance [34].

Our approach is based on fractional calculus [32], a well-known mathematical framework with a 176 broad range of applications [50,51]. In this framework, ecological memory is represented by fractional 177 derivatives and their associated kernel, which determines how quickly the influence of past states fades out 178 (see Appendix S1). Commensurate fractional derivatives have previously been shown to cause intrinsic 179 damping in a system [52-54], which may delay transitions or shift critical thresholds [33]. Incommensurate 180 models, on the other hand, yield complicated ODE systems that are mathematically more challenging 181 to analyse and therefore remain less well understood. We have shown here that the type of memory 182 introduced by fractional derivatives can influence resistance and resilience in ecological communities. 183 Quantifying this influence using recently proposed resilience measures, such as exit time [55], would 184 provide a promising line of research for future work. While this framework allows introducing only a 185 specific type of memory, our qualitative results on the influence of memory on community dynamics are 186 likely to hold more generally.

In addition to damping, memory can also induce other dynamical properties, such as long periods of 188 instability [36], or long transients [38], which have been reported in ecological systems [56] and chemostat 189 experiments [57]. Long transients have previously been shown to be favored by stochasticity, multiple 190 time scales, and high dimensionality [38], and our results indicate that memory should be added to this 191 list; [38] also argue that regime shifts may occur during such long transient dynamics, without requiring 192 parameter changes. Our results support this view, since we have shown that changes in incommensurate 193 memory can trigger abrupt regime shifts even in the absence of perturbations. 194

Modeling real systems using models that incorporate memory would benefit from the ability to gather 195 empirical evidence for the presence, strength, and quality of memory in the system. Recent literature 196 suggests that it might indeed be possible to empirically detect the presence of memory based on the broad 197 properties of a time series. It has been shown that longitudinal time series of microbial communities may 198 carry detectable signatures of underlying ecological processes [4,58]; and recently, Bayesian hierarchical 199 models [10,14], Random Forests [11], neural networks [59], and unsupervised Hebbian learning [60] have 200 been proposed to detect signatures of memory in other contexts.

Several extensions of our model could be considered in future studies to enhance its flexibility and 202 model memory more generally, such as varying initial times [33] or applying fractional differential equations 203 with time-varying derivative orders [61]. Alternative approaches have also been considered to model 204 ecological memory. These include the incorporation of autocorrelation or fixed time-lags into the model 205 structure [15]. One could also model ecological memory by distributed delay differential equations 206 (DDE) [62], fractional delay differential equations [63], or an integer memory-dependent derivative [64] 207 with arbitrary kernel functions to shape different patterns of memory weights. 
Ecological memory is a systemic property that can arise through various mechanisms. For instance, 209 communities can alter their environment and thus modify environmental parameters in ways that reflect 210 past events, or organisms may exhibit context-specific growth patterns that reflect adaptations $[60,65,66]$. 211 Delay effects could also arise without memory and through other mechanisms, such as intracellular inertia. 212 Species may indeed have different and often variable lag phases, due to complex intracellular processes 213 that may be effectively memoryless. In such cases, the dampening effects could be simply modeled by 214 introducing a "break" that would slow down or create a lag in community dynamics without inducing 215 actual memory effects. Specifically designed longitudinal experiments could help evaluate the types and 216 relative strengths of memory in real communities, such as in synthethic microbial communities that 217 can be used to collect long and dense time series with highly controlled perturbations and replicated 218 experiments.

Improving our understanding of the key mechanisms underlying community dynamics is a necessity 220 to generate more accurate predictions, and ultimately to develop new techniques for the manipulation of 221 complex ecological communities. We have combined theoretical analysis with computational simulations 222 to explore the various facets of the influence of ecological memory and highlighted its often overlooked 223 role as a key determinant of complex community dynamics.

\section{References}

1. Gonze D, Coyte KZ, Lahti L, Faust K. Microbial communities as dynamical systems. Curr Opin 226 Microbiol. 2018;44:41-49. doi:10.1016/j.mib.2018.07.004.

2. Quince C, Walker AW, Simpson JT, Loman NJ, Segata N. Shotgun metagenomics, from sampling 228 to analysis. Nat biotechnol. 2017;35(9):833-844. doi:10.1038/nbt.3935.

3. Song HS, Cannon WR, Beliaev AS, Konopka A. Mathematical Modeling of Microbial Community 230 Dynamics: A Methodological Review. Processes. 2014;2(4):711-752. doi:10.3390/pr2040711. 231

4. Faust K, Bauchinger F, Laroche B, De Buyl S, Lahti L, Washburne AD, et al. Signatures 232 of ecological processes in microbial community time series. Microbiome. 2018;6(1):1-13. 233 doi:10.1186/s40168-018-0496-2.

5. Coenen AR, Hu SK, Luo E, Muratore D, Weitz JS. A Primer for Microbiome Time-Series Analysis. 235 Front genet. 2020;11:310-310. doi:10.3389/fgene.2020.00310.

6. Björk JR, Dasari M, Grieneisen L, Archie EA. Primate microbiomes over time: Longitudinal 237 answers to standing questions in microbiome research. American Journal of Primatology. 238 2019;81(10-11):e22970. doi:10.1002/ajp.22970. 
7. Fukuyama J, Rumker L, Sankaran K, Jeganathan P, Dethlefsen L, Relman DA, et al. Multidomain 240 analyses of a longitudinal human microbiome intestinal cleanout perturbation experiment. PLoS 241 Comput Biol. 2017;13(8):1-29. doi:10.1371/journal.pcbi.1005706.

8. Gunderson LH. Ecological Resilience-in Theory and Application. Annu Rev Ecol Evol Syst. 243 2000;31(1):425-439. doi:10.1146/annurev.ecolsys.31.1.425.

9. Johnstone JF, Allen CD, Franklin JF, Frelich LE, Harvey BJ, Higuera PE, et al. 245 Changing disturbance regimes, ecological memory, and forest resilience. Front Ecol Environ. 246 2016;14(7):369-378. doi:10.1002/fee.1311.

10. Ogle K, Barber JJ, Barron-Gafford GA, Bentley LP, Young JM, Huxman TE, et al. 248 Quantifying ecological memory in plant and ecosystem processes. Ecol Lett. 2015;18(3):221-235. 249 doi:10.1111/ele.12399.

11. Benito BM, Gil-Romera G, Birks HJB. Ecological memory at millennial time-scales: the 251 importance of data constraints, species longevity and niche features. Ecography. 2020;43(1):1-10. 252 doi:10.1111/ecog.04772.

12. Schweiger AH, Boulangeat I, Conradi T, Davis M, Svenning JC. The importance of ecological 254 memory for trophic rewilding as an ecosystem restoration approach. Biol Rev. 2019;94(1):1-15. 255 doi:10.1111/brv.12432.

13. Žliobaitè I, Fortelius M, Stenseth NC. Reconciling taxon senescence with the Red Queen's hypothesis. 257 Nature. 2017;552(7683):92-95. doi:10.1038/nature24656.

14. Itter MS, Vanhatalo J, Finley AO. EcoMem: An R package for quantifying ecological memory. 259 Environ Model Softw. 2019;119:305-308. doi:10.1016/j.envsoft.2019.06.004.

15. Golinski M, Bauch C, Anand M. The effects of endogenous ecological memory on 261 population stability and resilience in a variable environment. Ecol Model. 2008;212(3):334-341. 262 doi:10.1016/j.ecolmodel.2007.11.005.

16. Schaefer V. Alien invasions, ecological restoration in cities and the loss of ecological memory. Restor 264 Ecol. 2009;17(2):171-176. doi:10.1111/j.1526-100X.2008.00513.x.

17. Bengtsson J, Angelstam P, Elmqvist T, Emanuelsson U, Folke C, Ihse M, et al. Reserves, resilience 266 and dynamic landscapes. Ambio. 2003;32(6):389-396. doi:10.1579/0044-7447-32.6.389.

18. Gonze D, Lahti L, Raes J, Faust K. Multi-stability and the origin of microbial community types. 268 ISME J. 2017;11(10):2159. doi:10.1038/ismej.2017.60. 
19. Gibson T, Gerber G. Robust and Scalable Models of Microbiome Dynamics. In: Proceedings of 270 the 35th International Conference on Machine Learning. vol. 80. PMLR; 2018. p. 1763-1772. 271

20. Marino S, Baxter NT, Huffnagle GB, Petrosino JF, Schloss PD. Mathematical modeling of primary 272 succession of murine intestinal microbiota. Proc Natl Acad Sci USA. 2014;111(1):439-444.

21. Stein RR, Bucci V, Toussaint NC, Buffie CG, Rätsch G, Pamer EG, et al. Ecological modeling from 274 time-series inference: insight into dynamics and stability of intestinal microbiota. PLoS Comput 275 Biol. 2013;9(12):e1003388. doi:10.1371/journal.pcbi.1003388.

22. Bucci V, Tzen B, Li N, Simmons M, Tanoue T, Bogart E, et al. MDSINE: Microbial Dynamical 277 Systems INference Engine for microbiome time-series analyses. Genome Biol. 2016;17(1):1-17. 278 doi:10.1186/s13059-016-0980-6.

23. Shenhav L, Furman O, Briscoe L, Thompson M, Silverman JD, Mizrahi I, et al. Modeling the 280 temporal dynamics of the gut microbial community in adults and infants. PLoS Comput Biol. 281 2019;15(6):e1006960. doi:10.1371/journal.pcbi.1006960.

24. Li C, Chng KR, Kwah JS, Av-Shalom TV, Tucker-Kellogg L, Nagarajan N. An 283 expectation-maximization algorithm enables accurate ecological modeling using longitudinal 284 microbiome sequencing data. Microbiome. 2019;7(1):1-14. doi:10.1186/s40168-019-0729-z. $\quad 285$

25. Joseph TA, Shenhav L, Xavier JB, Halperin E, Pe'er I. Compositional Lotka-Volterra 286 describes microbial dynamics in the simplex. PLoS Comput Biol. 2020;16(5):e1007917. 287 doi:10.1371/journal.pcbi.1007917.

26. McGeachie MJ, Sordillo JE, Gibson T, Weinstock GM, Liu YY, Gold DR, et al. Longitudinal 289 prediction of the infant gut microbiome with dynamic bayesian networks. Sci Rep. 2016;6:20359. 290 doi:10.1038/srep20359.

27. Ravel J, Gajer P, Abdo Z, Schneider GM, Koenig SSK, McCulle SL, et al. Vaginal microbiome 292 of reproductive-age women. Proc Natl Acad Sci USA. 2011;108(Supplement 1):4680-4687. 293 doi:10.1073/pnas.1002611107.

28. Falony G, Joossens M, Vieira-Silva S, Wang J, Darzi Y, Faust K, et al. Population-level analysis of 295 gut microbiome variation. Science. 2016;352(6285):560-564. doi:10.1126/science.aad3503.

29. Arumugam M, Raes J, Pelletier E, Le Paslier D, Yamada T, Mende DR, et al. Enterotypes of the 297 human gut microbiome. Nature. 2011;473(7346):174-180. doi:10.1038/nature09944.

30. Ding T, Schloss PD. Dynamics and associations of microbial community types across the human 299 body. Nature. 2014;509(7500):357-360. doi:10.1038/nature13178. 
31. Costea PI, Hildebrand F, Arumugam M, Bäckhed F, Blaser MJ, Bushman FD, et al. Enterotypes 301 in the landscape of gut microbial community composition. Nat Microbiol. 2018;3(1):8-16. 302 doi:10.1038/s41564-017-0072-8.

32. Du M, Wang Z, Hu H. Measuring memory with the order of fractional derivative. Sci Rep. 304 2013;3(1):1-3. doi:10.1038/srep03431.

33. Saeedian M, Khalighi M, Azimi-Tafreshi N, Jafari GR, Ausloos M. Memory effects on epidemic 306 evolution: The susceptible-infected-recovered epidemic model. Phys Rev E. 2017;95:022409. 307 doi:10.1103/PhysRevE.95.022409.

34. Marrec L, Bitbol AF. Resist or perish: Fate of a microbial population subjected to a periodic presence 309 of antimicrobial. PLoS Comput Biol. 2020;16(4):e1007798. doi:10.1371/journal.pcbi.1007798.

35. Rivero M, Rogosin SV, Tenreiro Machado JA, Trujillo JJ. Stability of fractional order systems. 311 Math Probl Eng. 2013;2013:356215. doi:10.1155/2013/356215.

36. Spanbauer TL, Allen CR, Angeler DG, Eason T, Fritz SC, Garmestani AS, et al. Prolonged 313 instability prior to a regime shift. PLoS One. 2014;9(10). doi:10.1371/journal.pone.0108936.

37. Tropini C, Moss EL, Merrill BD, Ng KM, Higginbottom SK, Casavant EP, et al. Transient osmotic 315 perturbation causes long-term alteration to the gut microbiota. Cell. 2018;173(7):1742-1754. 316 doi:10.1016/j.cell.2018.05.008.

38. Hastings A, Abbott KC, Cuddington K, Francis T, Gellner G, Lai YC, et al. Transient phenomena 318 in ecology. Science. 2018;361(6406):eaat6412. doi:10.1126/science.aat6412.

39. Amirian MM, Towers IN, Jovanoski Z, Irwin AJ. Memory and mutualism in species 320 sustainability: A time-fractional Lotka-Volterra model with harvesting. Heliyon. 2020;6(9):e04816. 321 doi:10.1016/j.heliyon.2020.e04816.

40. Khalighi M, Eftekhari L, Hosseinpour S, Lahti L. Three-species Lotka-Volterra model 323 with respect to Caputo and Caputo-Fabrizio fractional operators. Symmetry. 2021;13(3):368. 324 doi:10.3390/sym13030368.

41. Sommer F, Anderson JM, Bharti R, Raes J, Rosenstiel P. The resilience of the intestinal microbiota 326 influences health and disease. Nat Rev Microbiol. 2017;15(10):630-638. doi:10.1038/nrmicro.2017.58. 327

42. Khazaei T, Williams RL, Bogatyrev SR, Doyle JC, Henry CS, Ismagilov RF. Metabolic multistability 328 and hysteresis in a model aerobe-anaerobe microbiome community. Sci Adv. 2020;6(33):eaba0353. 329 doi:10.1126/sciadv.aba0353. 
43. Bush T, Diao M, Allen RJ, Sinnige R, Muyzer G, Huisman J. Oxic-anoxic regime shifts mediated 331 by feedbacks between biogeochemical processes and microbial community dynamics. Nat Commun. 332 2017;8(1):1-9. doi:https://doi.org/10.1038/s41467-017-00912-x.

44. Goyal A, Dubinkina V, Maslov S. Multiple stable states in microbial communities explained by 334 the stable marriage problem. ISME J. 2018;12(12):2823-2834. doi:10.1038/s41396-018-0222-x. 335

45. Hutchinson GE. The paradox of the plankton. Am Nat. 1961;95(882):137-145. doi:10.1086/282171. 336

46. Huston M. A general hypothesis of species diversity. Am Nat. 1979;113(1):81-101. 337 doi:10.1086/283366.

47. Abreu CI, Andersen Woltz VL, Friedman J, Gore J. Microbial communities display 339 alternative stable states in a fluctuating environment. PLoS Comput Biol. 2020;16(5):1-17. 340 doi:10.1371/journal.pcbi.1007934.

48. Cairns J, Jokela R, Becks L, Mustonen V, Hiltunen T. Repeatable ecological dynamics govern 342 the response of experimental communities to antibiotic pulse perturbation. Nat Ecol Evol. 343 2020;4(10):1385-1394. doi:10.1038/s41559-020-1272-9.

49. Hiltunen T, Virta M, Laine AL. Antibiotic resistance in the wild: an eco-evolutionary perspective. 345 Philos Trans R Soc Lond, B, Biol Sci. 2017;372(1712):20160039. doi:10.1098/rstb.2016.0039.

50. Sun H, Zhang Y, Baleanu D, Chen W, Chen Y. A new collection of real world applications of 347 fractional calculus in science and engineering. Commun Nonlinear Sci Numer Simul. 2018;64:213-231. 348 doi:10.1016/j.cnsns.2018.04.019.

51. Almeida R, Bastos NR, Monteiro MTT. Modeling some real phenomena by fractional differential 350 equations. Math Methods Appl Sci. 2016;39(16):4846-4855. doi:10.1002/mma.3818.

52. Achar BNN, Hanneken JW, Clarke T. Response characteristics of a fractional oscillator. Physica 352 A. 2002;309(3):275-288. doi:10.1016/S0378-4371(02)00609-X.

53. Tarasov VE. Quantum dissipation from power-law memory. Ann Phys (N Y). 2012;327(6):1719-1729. 354 doi:10.1016/j.aop.2012.02.011.

54. Tofighi A. The intrinsic damping of the fractional oscillator. Physica A. 2003;329(1):29-34. 356 doi:10.1016/S0378-4371(03)00598-3.

55. Arani BMS, Carpenter SR, Lahti L, van Nes EH, Scheffer M. Exit time as a measure of ecological 358 resilience. Science. 2021;372(6547). doi:10.1126/science.aay4895. 
56. Morozov A, Abbott K, Cuddington K, Francis T, Gellner G, Hastings A, et al. Long transients in 360 ecology: Theory and applications. Phys Life Rev. 2020;32:1-40. doi:10.1016/j.plrev.2019.09.004. 361

57. Fukami T. Assembly history interacts with ecosystem size to influence species diversity. Ecology. 362 2004;85(12):3234-3242. doi:10.1890/04-0340.

58. Faust K, Lahti L, Gonze D, de Vos WM, Raes J. Metagenomics meets time series analysis: unraveling 364 microbial community dynamics. Curr Opin Microbiol. 2015;25:56-66. doi:10.1016/j.mib.2015.04.004. 365

59. Armstrong McKay DI, Dyke JG, Doncaster CP, Dearing JA, Wang R. Network-based metrics of 366 resilience and ecological memory in lake ecosystems. bioRxiv. 2020;doi:10.1101/810762.

60. Power DA, Watson RA, Szathmáry E, Mills R, Powers ST, Doncaster CP, et al. What can ecosystems 368 learn? Expanding evolutionary ecology with learning theory. Biology Direct. 2015;10(1):69. 369 doi:10.1186/s13062-015-0094-1.

61. Sun H, Chang A, Zhang Y, Chen W. A review on variable-order fractional differential equations: 371 mathematical foundations, physical models, numerical methods and applications. Fract Calc Appl 372 Anal. 2019;22(1):27-59. doi:10.1142/S0218127412300145.

62. Cooke KL, Grossman Z. Discrete delay, distributed delay and stability switches. J Math Anal 374 Appl. 1982;86(2):592-627. doi:10.1016/0022-247X(82)90243-8.

63. Zúñiga-Aguilar C, Coronel-Escamilla A, Gómez-Aguilar J, Alvarado-Martínez V, Romero-Ugalde 376 H. New numerical approximation for solving fractional delay differential equations of variable order 377 using artificial neural networks. Eur Phys J Plus. 2018;133(2):1-16. doi:10.1140/epjp/i2018-11917-0. 378

64. Wang JL, Li HF. Memory-dependent derivative versus fractional derivative (I): Difference in 379 temporal modeling. J Comput Appl Math. 2021;384:112923. doi:10.1016/j.cam.2020.112923. 380

65. Miller AD, Inamine H, Buckling A, Roxburgh SH, Shea K. How disturbance history alters invasion 381 success: biotic legacies and regime change. Ecol Lett. 2021;24(4):687-697. doi:10.1111/ele.13685. 382

66. Yen P, Papin JA. History of antibiotic adaptation influences microbial evolutionary dynamics 383 during subsequent treatment. PLoS Biol. 2017;15(8):1-34. doi:10.1371/journal.pbio.2001586. 384

67. Safdari H, Kamali MZ, Shirazi A, Khalighi M, Jafari G, Ausloos M. Fractional dynamics 385 of network growth constrained by aging node interactions. PLoS One. 2016;11(5):e0154983. 386 doi:10.1371/journal.pone.0154983.

68. Li C, Zhang F. A survey on the stability of fractional differential equations. Eur Phys J Spec Top. 388 2011;193(1):27-47. doi:10.1140/epjst/e2011-01379-1. 
69. Emir K. Stability analysis of fractional differential equations with unknown parameters. Nonlinear 390 Anal Model Control. 2019;24(2):224-240. doi:10.15388/NA.2019.2.5.

70. Petráš I. Chaos in fractional-order population model. Int J Bifurc Chaos Appl Sci Eng. 392 2012;22(04):1250072. doi:10.1142/S0218127412500721.

71. de Oliveira EC, Tenreiro Machado JA. A Review of Definitions for Fractional Derivatives and 394 Integral. Math Probl Eng. 2014;2014. doi:10.1155/2014/238459.

72. Caputo M. Linear models of dissipation whose Q is almost frequency independent-II. Geophys J 396 Int. 1967;13(5):529-539. doi:10.1111/j.1365-246X.1967.tb02303.x.

73. Kilbas AA, Srivastava HM, Trujillo JJ. Theory and applications of fractional differential equations. 398 vol. 204. Elsevier; 2006.

74. Podlubny I. Fractional differential equations: An introduction to fractional derivatives, fractional 400 differential equations, to methods of their solution and some of their applications. Elsevier; 1998.401

75. Diethelm K, Ford NJ, Freed AD. A predictor-corrector approach for the numerical solution of 402 fractional differential equations. Nonlinear Dyn. 2002;29(1-4):3-22. doi:10.1023/A:1016592219341. 403

76. Garrappa R. On linear stability of predictor-corrector algorithms for fractional differential equations. 404 Int J Comput Math. 2010;87(10):2281-2290. doi:10.1080/00207160802624331.

77. Khalighi M. moeinkh88/Moein-Khalighi: article_2021_memoryeffects; 2021. Zenodo. Available 406 from: https://doi.org/10.5281/zenodo.4725218.

78. Diethelm K, Ford NJ, Freed AD. Detailed error analysis for a fractional Adams method. Numer 408 Algorithms. 2004;36(1):31-52. doi:10.1023/B:NUMA.0000027736.85078.be. 


\section{Supporting information}

\section{Appendix S1}

In the following, we detail the mathematical aspects of incorporating ecological memory into a non-linear 412 extension of the generalized Lotka-Volterra model.

We used as a starting point the following memoryless model, introduced by [18]:

$$
\begin{array}{r}
\frac{d X_{i}}{d t}=X_{i}\left(b_{i} f_{i}\left(\left\{X_{k}\right\}\right)-k_{i} X_{i}\right), \\
f_{i}\left(\left\{X_{k}\right\}\right)=\prod_{\substack{k=1 \\
k \neq i}}^{N} \frac{K_{i k}^{n}}{K_{i k}^{n}+X_{k}^{n}} .
\end{array}
$$

This model describes the dynamics of each microbial species abundance $X_{i}$ according to its growth rate 416 $b_{i}$, its death rate $k_{i}$ and an inhibition term $f_{i}$, which is defined by interaction constants $K_{i j}$ and the Hill 417 coefficient $n$ as parameters. $K_{i j}$ represents the inhibition of species $i$ by species $j$ : the lower it is, the 418 stronger the inhibition.

The interaction matrix $\mathbf{K}=\left\{K_{i j}\right\}$ was generated based on two alternative approaches. The first 420 approach allocates the predefined species in three groups (see below and Fig 5 as in [18]), thereby setting 421 different values of inter-group versus intra-group interactions. The second approach does not impose a 422 predefined structure for the interaction matrix K (Fig S4).

In the three-group approach, we define three sets of species indices by B (blue), R (red), and G (green). 425 Each species $i$ belongs to exactly one of these three groups. We define the growth rate of each group by 426 the growth vector $\mathbf{b}=\left[b_{B}, b_{R}, b_{G}\right]$, where $b_{B}=\left\{b_{i} \mid i \in B\right\}, b_{R}=\left\{b_{i} \mid i \in R\right\}$, and $b_{G}=\left\{b_{i} \mid i \in G\right\} .427$ We also define the interaction matrix $\mathbf{K}=\left\{K_{i j} \mid i, j \in B\right.$ or $R$ or $\left.G\right\}$ such that $K_{i j}$ only depends on 428 the group memberships of species $i$ and $j$, up to a slight noise (see Fig 5a and Appendix S2). We first 429 considered a community of three species (i.e., only one species per group), and then a community of 15430 species forming three groups with strong inter-group inhibition and weak intra-group inhibition.

If the inhibition strength is large enough ( small $K_{i j}$ ), this model can have three coexisting stable 432 states (tristability). This tristable community is dominated by either one of its three groups depending 433 on initial species abundances, interaction matrix $\mathbf{K}$, and growth vector $\mathbf{b}$. 


\section{Incorporating memory by fractional calculus}

Fractional order derivatives have been successfully used to account for memory effects in many disciplines [32, 436 33,67]. This approach requires defining a temporal kernel in dynamical models [32,33]. The stable regions 437 of fractional differential equations differ from the corresponding classical one $[35,68,69]$ and thus induce 438 significant differences in the stability landscape of a community model. Interestingly, chaos has been 439 observed in a fractional population model [70], which exhibits a structure entirely different from typical 440 dynamical attractors such as the Rössler or Lorenz attractors.

To introduce memory in ODE models, we replace the ordinary time derivative in system (1) by the 442 fractional derivative $\mathfrak{D}^{\mu_{i}}$. This leads to the appearance of a time correlation function (a memory kernel) 443 which imposes a dependency between the current system state and its past trajectory. The past states of 444 the system influence the current dynamics, giving rise to memory effects.

Let us now rewrite the initial model in (1) by employing fractional derivatives and the simplifying 446 notation $F_{i}=F\left(t, X_{i}\right):=X_{i}\left(b_{i} f_{i}\left(\left\{X_{k}\right\}\right)-k_{i} X_{i}\right)$, as:

$$
\mathfrak{D}^{\mu_{i}} X_{i}=F_{i}, \quad 0<\mu_{i} \leq 1, \mu_{i} \in \mathbb{R}
$$

There are different definitions of fractional time derivatives for different purposes [71]. We use here the 448 Caputo fractional time derivative [72], $\mathfrak{D}^{\mu_{i}}:={ }_{t_{0}}^{c} D_{t}^{\mu_{i}}$, as a control parameter of memory effects because 449 of its intuitive interpretation. This derivative is defined by the following integral equation for a given 450 function $g(t)$ :

$$
{ }_{t_{0}}^{c} D_{t}^{\mu} g(t)={ }_{t_{0}} I_{t}^{1-\mu} g^{\prime}(t)=\frac{1}{\Gamma(1-\mu)} \int_{t_{0}}^{t} \frac{g^{\prime}(\tau) d \tau}{(t-\tau)^{\mu}}, \quad 0<\mu \leq 1
$$

in which ${ }_{t_{0}} I_{t}^{1-\mu}$ is the fractional integral of order $1-\mu$ that is defined by

$$
{ }_{t_{0}} I_{t}^{\mu} g(t)=\frac{1}{\Gamma(\mu)} \int_{t_{0}}^{t} \frac{g(\tau) d \tau}{(t-\tau)^{1-\mu}}
$$

where $\Gamma$ denotes the gamma function. Throughout this article, we quantify memory as $1-\mu$.

To provide an intuitive interpretation of the new system equation (2), let us apply a fractional Caputo 455 derivative of order $1-\mu_{i}$ on both sides of (2):

$$
{ }_{t_{0}}^{c} D_{t}^{1-\mu_{i}}\left({ }_{t_{0}}^{c} D_{t}^{\mu_{i}} X_{i}\right)={ }_{t_{0}}^{c} D_{t}^{1-\mu_{i}}\left(F_{i}\right)
$$


Because the Caputo fractional derivatives of order $\mu$ and $-\mu$ are inverse operators [73, 74], this simplifies

as:

$$
{ }_{t_{0}}^{c} D_{t}^{1} X_{i}=\frac{d X_{i}}{d t}={ }_{t_{0}}^{c} D_{t}^{1-\mu_{i}}\left(F_{i}\right)
$$

Equation (6) shows that for $\mu_{i}=1$ we retrieve the standard integer derivative model (1) as a special 459 case of the fractional derivative model (2), since the fractional operator becomes the unity operator for a 460 fractional order of 1. Furthermore, the right-hand side of equation (6) can be expressed as the fractional 461 integral of order $\left(\mu_{i}-1\right)$ on the interval $\left[t_{0}, t\right]$, that is:

$$
\frac{d X_{i}(t)}{d t}=\frac{1}{\Gamma\left(\mu_{i}-1\right)} \int_{t_{0}}^{t}(t-\tau)^{\mu_{i}-2} F\left(\tau, X_{i}\right) d \tau
$$

The system described by equation (7) is a transformation of the original system (1) with an additional 463 memory contributions $\mu_{i}$. When $0<\mu_{i}<1$, the time-dependent memory kernel $\frac{1}{\Gamma\left(\mu_{i}-1\right)}(t-\tau)^{\mu_{i}-2} \quad 464$ guarantees the existence of temporal scaling behaviors which are common in nature. The memory kernel's 465 decay rate depends on $\mu_{i}$ : the lower the value of $\mu_{i}$, the slower it will decay. This shows how imposing 466 memory on the system equation (1) slows down community dynamics.

The derivative order $\mu_{i}$ can be used to control the strength of the memory so that when $\mu_{i}$ goes 468 toward the integer value 1 , the influence of memory decreases, and the system tends toward a Markov 469 process. In the context of microbial communities, memory may thus counteract the effects of species 470 interactions. In the memoryless case $\left(\mu_{i}=1\right)$, the kernel becomes a Dirac delta function, $\delta(t-\tau)$, which 471 results in the integer-order integrodifferential equation of model (1).

In summary, the Caputo fractional derivative provide a means to incorporate ecological memory in a 473 dynamical system based on a convolution integral with a power-law memory kernel. Besides, it could be 474 modified by a time-delay reflecting the duration of memory effects and the kernel function shaping the 475 memory weight [64].

\section{Numerical simulations}

Adams methods provide commonly used numerical solutions for ODEs, involving implicit (Adams-Moulton) 478 and explicit (Adams-Bashforth) linear multi-step schemes. We exploited in this paper the predictor-corrector 479 method based on Adams formulae (see [75,76]) and implemented it in MATLAB. The corresponding 480 code is available on Zenodo [77].

Given the system equation (2), let us write $\mathbf{X}$ the set of all species abundances, $\boldsymbol{\mu}$ the corresponding 482 vector of derivative orders $\mu_{i}$, and $\mathbf{F}$ the corresponding matrix function of all $X_{i}\left(b_{i} f_{i}\left(\left\{X_{k}\right\}\right)-k_{i} X_{i}\right)$. We 483 can then rewrite the fractional order model (2) in the following matrix form: 
The initial value problem (8) is equivalent to the Volterra integral equation $[73,75]$ :

$$
\mathbf{X}(t)=\mathbf{X}_{0}+\frac{1}{\Gamma(\boldsymbol{\mu})} \int_{t_{0}}^{t}(t-\tau)^{\boldsymbol{\mu}-1} \mathbf{F}(\tau, \mathbf{X}(\tau)) d \tau
$$

We solved Eq. (9) using a product integration technique, in which we replaced the function $\mathbf{F}(\tau, \mathbf{X}(\tau)) \quad 486$ with piece-wise interpolating polynomials. For the grid nodes $t_{j}(j=0, \ldots, m)$ with constant step size $h \quad 487$ $\left(t_{j}=t_{0}+j h\right)$, we write $\mathbf{F}_{j}=\mathbf{F}\left(t_{j}, \mathbf{X}_{j}\right)$ where $\mathbf{X}_{j}$ is the numerical approximation to $\mathbf{X}\left(t_{j}\right)$. The product 488 rectangle rule [75] gives an explicit estimation of Eq. (9) as a predictor:

$$
\begin{gathered}
\mathbf{X}_{m}=\mathbf{X}_{0}+h^{\boldsymbol{\mu}} \sum_{j=0}^{m-1} \mathbf{b}_{m-j-1} \mathbf{F}_{j} \\
\mathbf{b}_{m-j-1}=\frac{(m-j)^{\mu}-(m-j-1)^{\mu}}{\Gamma(\boldsymbol{\mu}+1)}
\end{gathered}
$$

and the product trapezoidal rule [75] provides an implicit estimation of Eq. (9) as a corrector:

$$
\begin{gathered}
\mathbf{X}_{m}=\mathbf{X}_{0}+h^{\boldsymbol{\mu}} \mathbf{c}_{m} \mathbf{F}_{0}+h^{\boldsymbol{\mu}} \sum_{j=1}^{m} \mathbf{d}_{m-j} \mathbf{F}_{j}, \\
\mathbf{c}_{m}=\frac{(m-1)^{\boldsymbol{\mu}+1}-m^{\mu}(m-\boldsymbol{\mu}-1)}{\Gamma(\boldsymbol{\mu}+2)}, \\
\mathbf{d}_{m-j}= \begin{cases}\frac{1}{\Gamma(\boldsymbol{\mu}+2)}, & \text { if } m-j=0, \\
\frac{(m-j-1)^{\mu+1}-2(m-j)^{\boldsymbol{\mu}+1}+(m-j+1)^{\mu+1}}{\Gamma(\boldsymbol{\mu}+2)}, & \text { if } m-j=1,2, \ldots\end{cases}
\end{gathered}
$$

The last term of the sum in the corrector equation (11), $\mathbf{F}\left(t_{m}, \mathbf{X}_{m}\right)$, is obtained by an approximation of 491 $\mathbf{X}_{m}$ in the predictor equation (10). This method is called FracPECE: Fractional Predict-Evaluate-Correct-Evadqeate [75]. Because its standard implementation was not sufficient considering the stiffness of the equation, we 493 improved its accuracy via an advanced convolution quadrature [76], and via multiple applications of the 494 corrector step [78] when required. Specifically, we used several corrector iterations when the difference 495 between two consecutive iterations was larger than the desired tolerance of $10^{-6}$. We considered a time 496 step size of $h=0.01$ or 0.005 for all simulations. 497

Note that since the model with fractional order derivatives (2) includes the standard model (1) as a 498 particular case (namely, for integer derivative order), the numerical approximations (10) and (11) are also 499 solutions to equation (1). The explicit solution (10)-or an assessment of the implicit solution (11)-shows 500 how memory influences the fundamental system dynamics through the dependence on $\mu$. 
bioRxiv preprint doi: https://doi.org/10.1101/2021.09.01.458486; this version posted September 2, 2021. The copyright holder for this preprint (which was not certified by peer review) is the author/funder, who has granted bioRxiv a license to display the preprint in perpetuity. It is made available under aCC-BY 4.0 International license.

\section{Appendix S2}

We provide in the Table below the detailed conditions and parameter values used in each of the numerical 503 experiments presented in the main text. Additional methodological clarifications for figures $5 \mathrm{c}$ and S1 are 504 given in the text below the table.

505

Table . Exact model specifications for the 3-species and 15-species systems.

\begin{tabular}{|c|c|c|c|c|c|c|c|c|c|c|c|c|}
\hline \multirow[t]{2}{*}{ Figure } & \multicolumn{3}{|c|}{$X_{0}$} & \multirow{2}{*}{$\begin{array}{c}K_{i j} \\
\forall i \neq j\end{array}$} & \multirow[t]{2}{*}{$n$} & \multirow{2}{*}{$\begin{array}{l}k_{i} \\
\forall i\end{array}$} & \multirow{2}{*}{\multicolumn{3}{|c|}{$\mathrm{B}=\stackrel{\mu}{\mathrm{R}=\mathrm{G}}$}} & \multicolumn{3}{|c|}{$b$} \\
\hline & B & $\mathrm{R}$ & G & & & & & & & B & $\mathrm{R}$ & $\mathrm{G}$ \\
\hline $\begin{array}{l}2 \mathrm{a} \\
2 \mathrm{~b} \\
3 \mathrm{a} \\
3 \mathrm{~b}\end{array}$ & 0.99 & 0.01 & 0.01 & 0.1 & 2 & 1 & $\begin{array}{ll} & \\
1 \& \\
1 \&\end{array}$ & $\begin{array}{l}\& 0 . \\
\& 0 . \\
0.9 \& \\
0.96 \&\end{array}$ & $\begin{array}{r} \\
.85 \\
0.9\end{array}$ & $\begin{array}{c}\text { Pulse1 } \\
\text { Pulse2 } \\
\text { Pulse3 } \\
\text { Periodic }\end{array}$ & 0.95 & $\begin{array}{c}\text { Pulse1 } \\
\text { Pulse2 } \\
1.05 \\
1.05\end{array}$ \\
\hline \multirow[t]{2}{*}{$4 b-c$} & $1 / 3$ & $1 / 3$ & $1 / 3$ & 0.1 & 2 & 1 & $\begin{array}{l}0 . \\
0 . \\
0 .\end{array}$ & $\begin{array}{c}1 \\
91579 \\
91579 \\
91579 \\
0.9 \\
0.7 \\
0.6\end{array}$ & & & chastic & \\
\hline & & & & & & & B & $\mathrm{R}$ & G & & & \\
\hline S1 & \multicolumn{3}{|c|}{ Equilibrium points } & $\begin{array}{l}\text { Random } \\
\text { interactions }\end{array}$ & 4 & 2 & \multicolumn{3}{|c|}{$\begin{array}{c}\text { No Specified Groups } \\
\mu_{i, \forall i}=1 \text { (or } 0.7 \text { ) }\end{array}$} & \multicolumn{3}{|c|}{$\begin{array}{l}\mathcal{N}(1,0.0025) \\
\text { with a pulse }\end{array}$} \\
\hline S2a & 0.99 & 0.01 & 0.01 & 0.1 & 2 & 1 & $\begin{array}{l}1 \\
1 \\
1\end{array}$ & $\begin{array}{l}1 \\
1 \\
1\end{array}$ & $\begin{array}{c}1 \\
0.90895 \\
0.90893\end{array}$ & Pulse4 & 0.95 & 1.05 \\
\hline $\mathrm{S} 2 \mathrm{~b}$ & $1 / 3$ & $1 / 3$ & $1 / 3$ & 0.1 & 2 & 1 & $\begin{array}{l}1 \\
1 \\
1\end{array}$ & $\begin{array}{c}1 \\
1 \\
0.9\end{array}$ & $\begin{array}{c}1 \\
0.8 \\
1\end{array}$ & & chastic & \\
\hline 5 & Unif & $r m(0,0.1)$ & & $\begin{array}{l}\text { Predefined } \\
\text { interactions }\end{array}$ & 2 & 1 & $\begin{array}{c}1 \\
0.6 \\
0.851841 \\
0.851840 \\
0.8\end{array}$ & 1 & 1 & & $1,0.01)$ & \\
\hline$S 3 \& \begin{array}{l}6 a \\
6 b\end{array}$ & {$[0.005,0.05]$} & $0.1 \& 0.3$ & $1 \& 0.1$ & 0.1 & 2 & 1 & $\begin{array}{c}1 \\
0.6\end{array}$ & $\begin{array}{c}1 \\
0.6\end{array}$ & $\begin{array}{l}1 \\
1\end{array}$ & 4 & 0.95 & 1.05 \\
\hline $\mathrm{S} 4$ & 0.25 & 0.25 & 0.25 & 0.2 & 2 & 0.5 & $\begin{array}{c}1 \\
1 \\
0.5\end{array}$ & $\begin{array}{c}1 \\
0.4 \\
0.6\end{array}$ & $\begin{array}{c}1 \\
0.7 \\
1\end{array}$ & & $1,0.01)$ & \\
\hline
\end{tabular}

Pulse1: $b_{B}(t)=0.5$ and $b_{G}(t)=2$ if $20 \leq t<60$, otherwise $b_{B}(t)=1$ and $b_{G}(t)=1.05$.

Pulse2: $b_{B}(t)=0.5$ and $b_{G}(t)=2.2$ if $20 \leq t<60$, otherwise $b_{B}(t)=1$ and $b_{G}(t)=1.05$.

Pulse3: $b_{B}(t)=0.2$ if $60 \leq t<100, b_{B}(t)=4.5$ if $200 \leq t<330$, otherwise $b_{B}(t)=1$.

Periodic: $b_{B}(t)=1$ if $20(2 m-2) \leq t<20(2 m-1), b_{B}(t)=0.2$ if $20(4 m-3) \leq t<20(4 m-2)$, $b_{B}(t)=4.5$ if $20(4 m-1) \leq t<20(4 m)$ where $m \in \mathbb{N}$.

Stochastic: The growth rates of these panels are generated by mean-reverting the Ornstein-Uhlenbeck Process described by the stochastic equation $d b_{t}=\theta\left(\phi-b_{t}\right) d t+\sigma d W_{t}$.

Random interactions: $K_{i j}=1-e^{-5 z}$, where $\mathrm{z}$ is randomly generated from a uniform distribution between 0 and 1.

Predefined interactions: $K_{i j} \sim 1+\mathcal{N}(0,0.01)$ for species $i$ and $j$ in the same group (intra-group interactions $\left.K_{B B}, K_{R R}, K_{G G}\right)$, and $K_{i j} \sim 0.5+\mathcal{N}(0,0.01)$ for species $i$ and $j$ in different groups (inter-group interactions).

Pulse $4: b_{B}(t)=0.2$ if $60 \leq t<100, b_{B}(t)=4.5$ if $400 \leq t<530$, otherwise $b_{B}(t)=1$. 
Fig 5c. Ternary plots allow representing the state of a 3-species or 3-group system by a single dot 506 and therefore are a convenient way to display the outcome of many simulations at a time. In Fig 5c, 507 each ternary plot shows the stable state distribution of the group relative abundances obtained for 50508 different simulations, each represented by a dot of the color of the dominant group. We detail below how 509 we computed the position of each dot in the triangle. Let us write $B, G$ and $R$ the average stable state

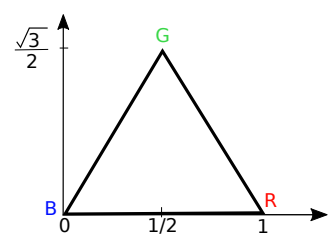

Fig . Triangle coordinates.

relative abundances of the species in the blue, green and red groups, that is $R=\frac{\sum_{i=1}^{5} R_{i}(\text { end })}{\sum_{i}\left(R_{i}+B_{i}+G_{i}\right)(\text { end })} \quad$ 511 (and similarly for $B$ and $G$ ), where $Z_{i}($ end) denotes the abundance of species $i$ in group $Z$ at the end of 512 the simulation. Let us consider an equilateral triangle in which each vertex corresponds to the complete 513 dominance of one group of species, as shown in the Figure above. Thus, a point (dot) close to the middle 514 of the triangle indicates a state of the system characterized by relatively even species abundances. If 515 $B=1(100 \%)$ is placed at $(x, y)=(0,0)$ and $R=1(100 \%)$ at $(1,0)$, then $G=1(100 \%)$ is at $\left(\frac{1}{2}, \frac{\sqrt{3}}{2}\right), 516$ and any triplet $(B, R, G)$ will be at $\left[\frac{1}{2}(2 R+G), \frac{\sqrt{3}}{2} G\right]$. These Cartesian coordinates provide a way to 517 map any triplet of group relative abundances to a unique location on the triangle.

Fig S1. Here, we randomly generated an interaction matrix $\mathbf{K}$ without predefined structure between 519 $N=15$ species. Specifically, we set $n=4$ and $K_{i j}=1-e^{-5 z}$, where $z$ is a randomly generated number 520 from a uniform distribution between 0 and 1 . We generated 10 communities, each with a random 521 vector of growth rates generated as $b_{i} \sim \mathcal{N}(1,0.0025), \forall i$. We used the same interaction matrix for all 522 10 communities, and death rates $k_{i}=2, \forall i$. For each community, we set the initial values for species 523 abundances $X_{i}$ at one of the equilibrium points of the system (randomly chosen). To compute the 524 dissimilarity of the community between times $t_{r}$ and $t_{p}$, we used the Bray-Curtis distance, computed as 525 $B C\left(t_{r}, t_{p}\right)=\frac{\sum_{i=r}^{N}\left|X_{i}\left(t_{r}\right)-X_{i}\left(t_{p}\right)\right|}{\sum_{i=1}^{N} X_{i}\left(t_{r}\right)+X_{i}\left(t_{p}\right)}$ 
bioRxiv preprint doi: https://doi.org/10.1101/2021.09.01.458486; this version posted September 2, 2021. The copyright holder for this
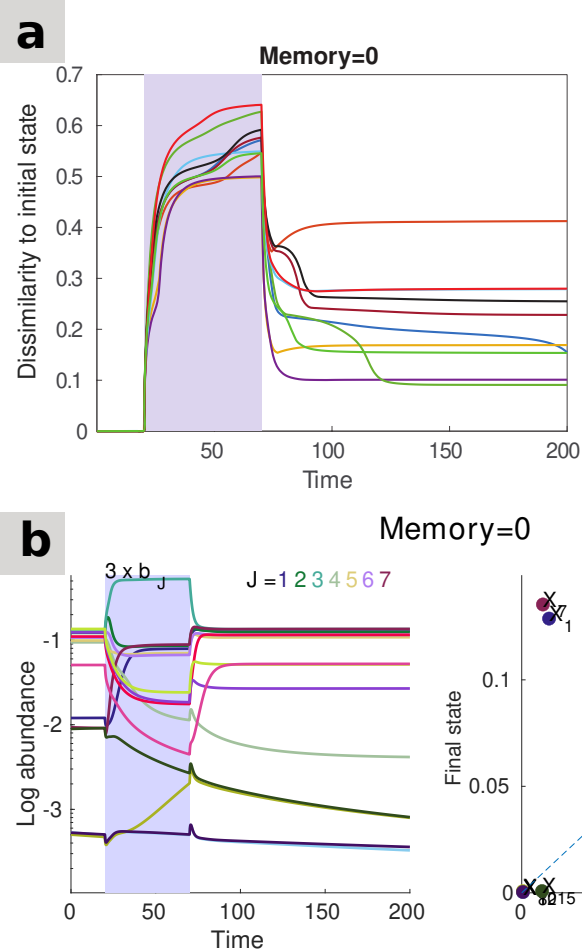

Memory $=0$

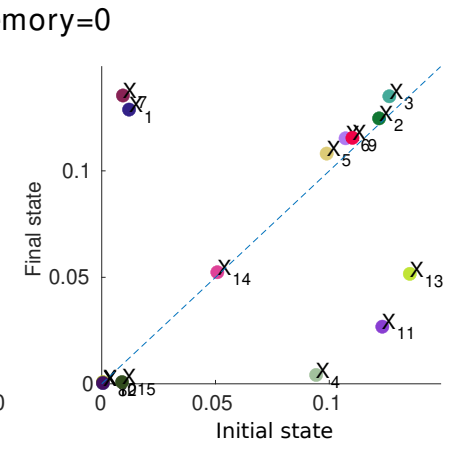

Memory $=0.7$
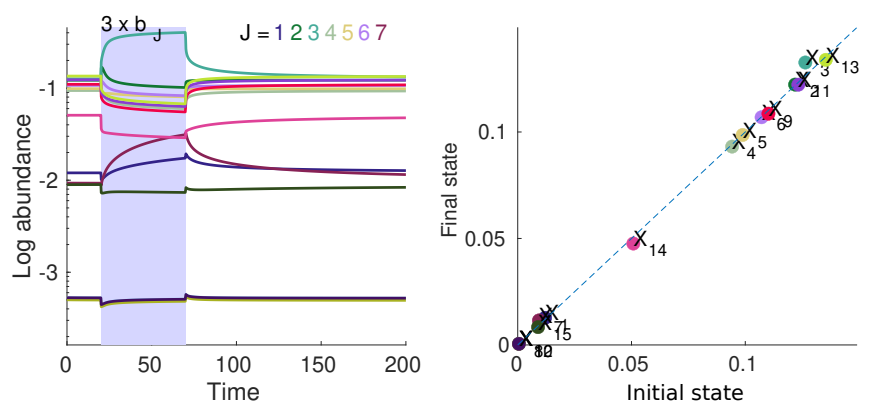
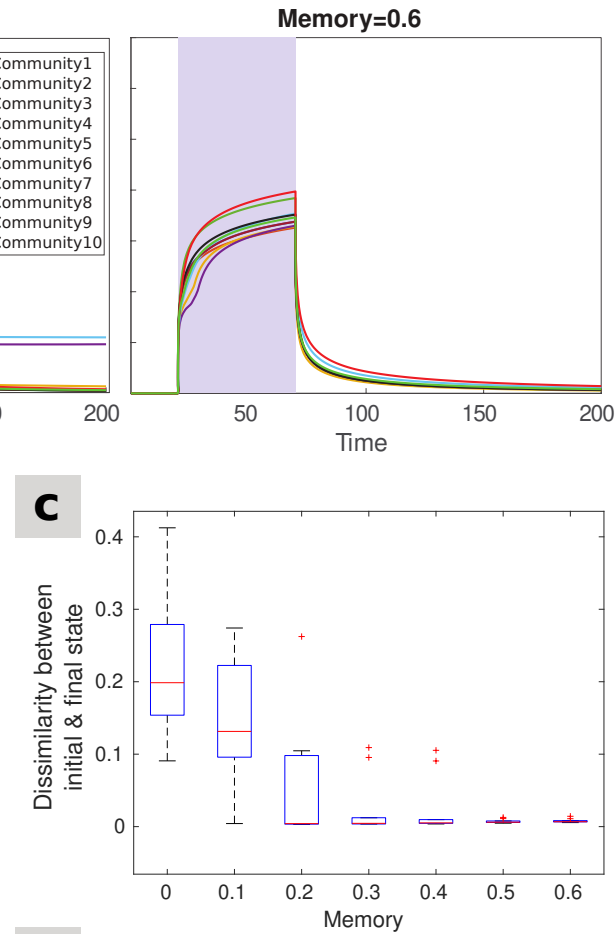

d

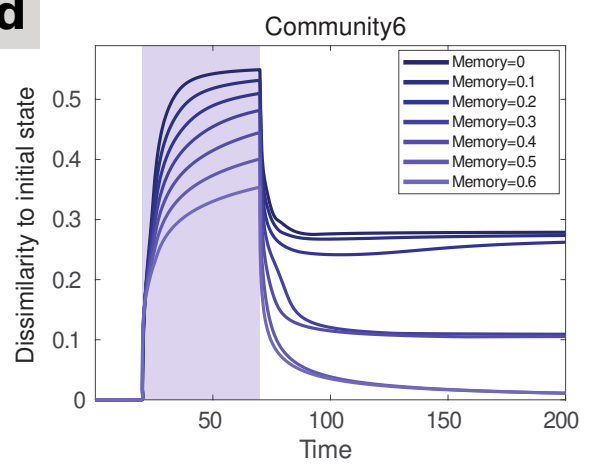

Fig S1. Memory effects preserve the stable state. We simulated ten communities of 15 species, each with random interaction matrices. A similar level of commensurate memory is applied to all ten communities. Every community is initially in a stable state of the system, and a perturbation is imposed by multiplying the growth rates of half of the species $\left(b_{1}, \ldots, b_{7}\right)$ by 3 . The simulation is stopped when the system is close to its new stable state. Although only the effect of commensurate memory is illustrated here, the same outcome can be achieved using incommensurate memory. (a) Dissimilarity to the initial stable state through time for all ten communities, for three different memory strengths. The stronger the memory, the more constrained the community trajectories are, and the more likely they are to revert to their initial stable state eventually. (b) Time series for one randomly chosen community, community 6 . The pulse perturbations lead the community to an alternative stable state in the absence of memory (top), while adding memory effects allows recovering the original state (bottom). (c) Community dissimilarity (Bray-Curtis) between the start and the end of the simulation for all ten communities and different memory levels. Without memory, the pulse perturbation changes the abundances of some of the species and leads to an alternative stable state (i.e., non-zero dissimilarity between start and end). In contrast, all communities recover their pre-perturbation stable state in the presence of memory (i.e., zero dissimilarity). (d) Dissimilarity to the initial stable state through time in community 6 , for different memory strengths. 
a Pulse

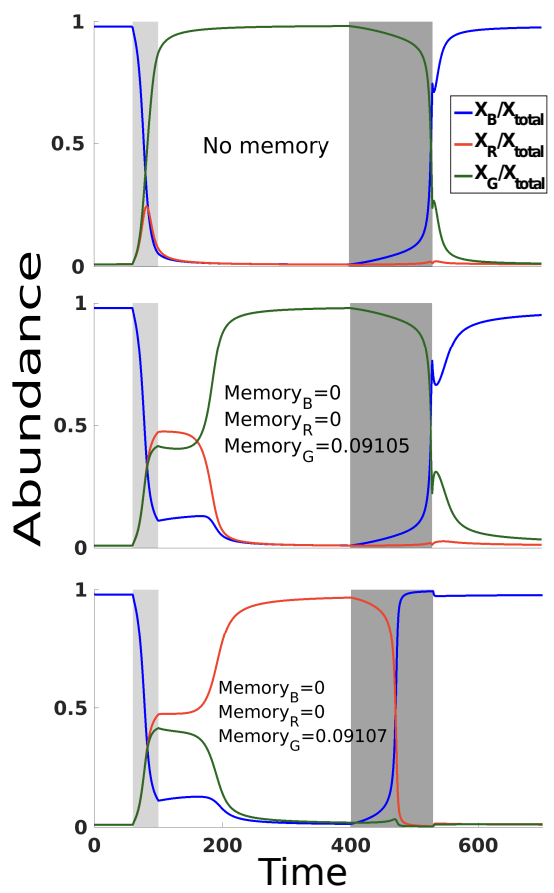

b Stochastic

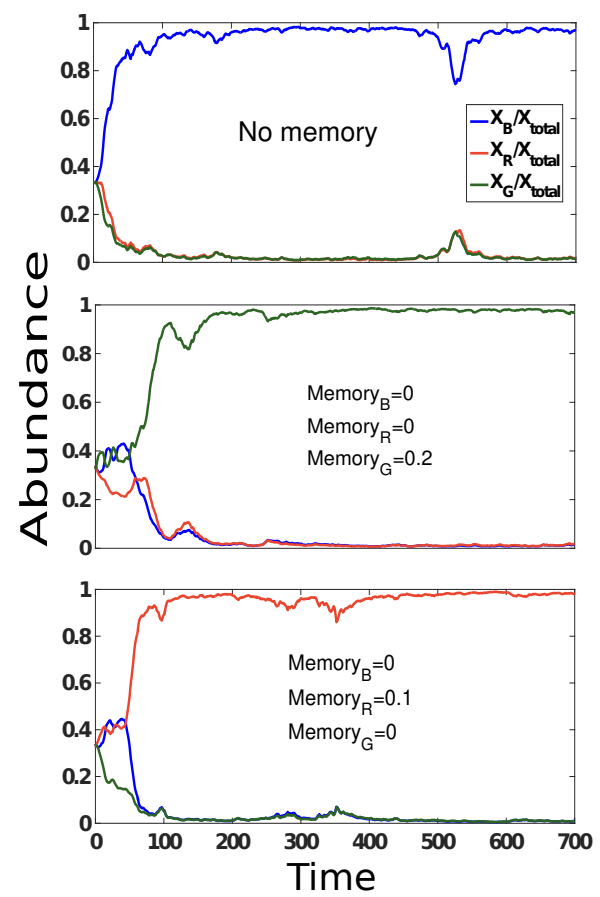

Fig S2. Role of memory in an incommensurate system. (a) The order derivative of $X_{G}$ is a non-integer value, $0<\mu_{G}<1$, and the derivative order of $X_{B}$ and $X_{R}$ are integer, $\mu_{B}=\mu_{R}=1$. Around a particular value of the order derivative of the green species $\left(\mu_{G}=0.90895\right)$, the system behaves differently after the first perturbation: for $\mu_{G}$ in the interval $\left.] 0.90895,1\right]$, the green species will be dominant, while in the interval $] 0,0.90894$ ] the red species will be dominant. (b) The growth rates follow the same Ornstein-Uhlenbeck process as in Fig 4. For the system without memory, the blue species is dominant. However, when imposing sufficient memory on the green or red species, they become dominant in the stable state. 

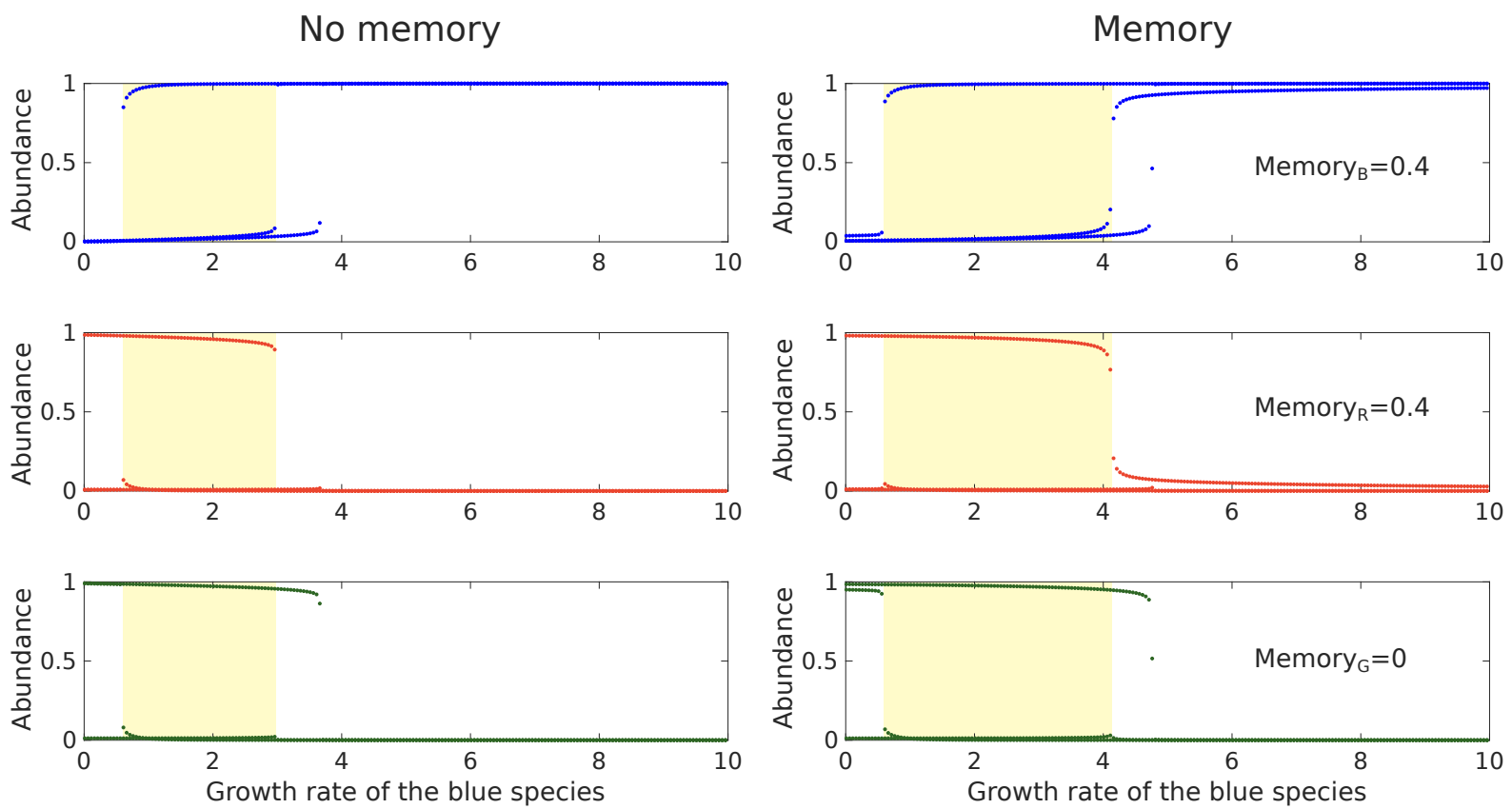

Fig S3. Memory broadens the model's multistable region. Bifurcation diagrams for the system shown in Fig 6, showing the relative abundance of the blue, red, and green species (from top to bottom) as a function of the blue species' growth rate for three different initial conditions (leading to three distinct curves per plot), in the absence (left) or presence (right) of memory. The yellow area shows the region of the parameter space that exhibits multistability, which is extended by the introduction of incommensurate memory (right).
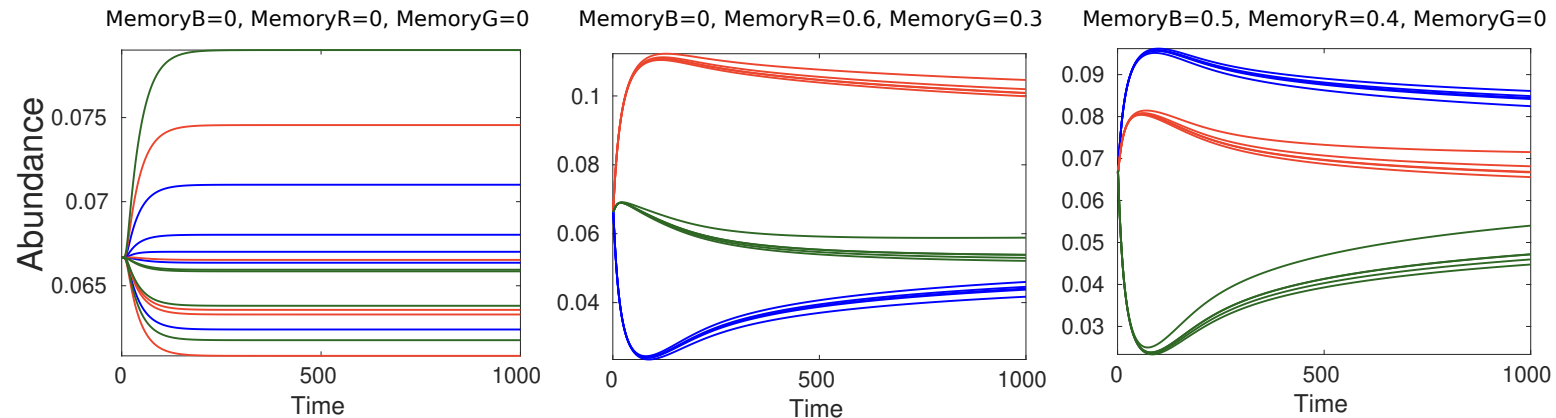

Fig S4. Groups of species can emerge from incommensurate memory. We consider here a 15-species community with equal interactions between species and identical initial abundances for all species, where species growth rates are drawn from $\mathcal{N}(1,0.01)$. As expected, the species do not form groups in the absence of memory (left panel). When the species are randomly split into three groups (red, green, blue) with varying degrees of memory, species with a similar degree of memory tend to exhibit similar dynamics and group together due to their shared memory properties (middle panel). A switch to an alternative stable state can be triggered by changing the strength of memory effects (right panel). 